NBER WORKING PAPER SERIES

\title{
BANKABLE PRICES
}

Garth Heutel

Working Paper 25235

http://www.nber.org/papers/w25235

\author{
NATIONAL BUREAU OF ECONOMIC RESEARCH \\ 1050 Massachusetts Avenue \\ Cambridge, MA 02138 \\ November 2018
}

Thanks to Billy Pizer, Marty Weitzman, and seminar participants at GSU for comments and to Puneet Arora for research assistance. The views expressed herein are those of the author and do not necessarily reflect the views of the National Bureau of Economic Research.

NBER working papers are circulated for discussion and comment purposes. They have not been peer-reviewed or been subject to the review by the NBER Board of Directors that accompanies official NBER publications.

(C) 2018 by Garth Heutel. All rights reserved. Short sections of text, not to exceed two paragraphs, may be quoted without explicit permission provided that full credit, including $\bigcirc$ notice, is given to the source. 
Bankable Prices

Garth Heutel

NBER Working Paper No. 25235

November 2018

JEL No. D62,H23,Q54,Q58

\begin{abstract}
$\underline{\text { ABSTRACT }}$
Allowing emissions permits to be banked and borrowed over time can yield efficiency gains. I develop a model to demonstrate that banking and borrowing can also be allowed for a price policy. I compare expected welfare between price and quantity policies, with and without banking, under several different scenarios regarding uncertainty. A bankable policy can provide an efficiency improvement by allowing for smoothing of costs, though it does not necessarily dominate a policy that does not allow banking. The ranking of prices vs. quantities and of bankability vs. non-bankability depends on both the slopes of marginal costs and benefits and on the specification of uncertainty.
\end{abstract}

Garth Heutel

436 Andrew Young School

Department of Economics

Georgia State University

PO Box 3992

Atlanta, GA 30302-3992

and NBER

gheutel@gsu.edu 


\section{Introduction}

Under perfect information, an externality market failure can be efficiently solved using either a price or a quantity instrument. For example, pollution can be reduced to its efficient level through either a pollution tax or a cap-and-trade scheme. Under certain types of information asymmetry, the equivalence between price and quantity instruments breaks down (Weitzman 1974). It has been argued that an advantage of quantity instruments over price instruments is that quantity instruments are bankable. For example, pollution permits in a capand-trade scheme can be banked for future use if abatement costs are lower than expected in the current period. Many real-world tradable pollution permit markets, like the Regional Greenhouse Gas Initiative and the European Union Emissions Trading Scheme, allow for banking or borrowing (Chevallier 2012).

Several papers that extend the framework of Weitzman (1974) consider the use of banking and borrowing of permits and its effect on efficiency. Fell et al. (2012) evaluate the welfare effects of allowing limited banking and borrowing, and they numerically simulate to find that allowing for banking and borrowing can make a quantity policy nearly as effective as a price policy. Williams (2002) argues that banking is optimal for stock but not flow pollutants. Kollenberg and Taschini (2016) model a policy in which the emissions cap is updated by policymakers in response to the number of permits in the bank to reduce costs. Most closely related to this study are two papers that use two-period models to study the efficiency of allowing banking. Pizer and Prest (2016) show that banking can improve efficiency and in some cases achieve the first best; by contrast Weitzman (2018) shows that banking is always dominated by either non-bankable ("fixed") quantities or non-bankable prices. 
The purpose of this paper is to consider another policy option that has until now been ignored by policy modelers: what I call bankable prices. Intertemporal trading, or bankability, can be built into a price policy as well as into a quantity policy. The firm is given a price in each period but can choose to defer payment for its actual output or emissions until the future - that is, the firm can bank some of its quantity in order to pay the price on it in a future period. Similarly, the firm can borrow forward, with no restrictions. The firm has the option of deferring or accelerating its tax liability. I develop a model allowing for bankable prices, and I compare that policy to three other policies: bankable quantities, non-bankable quantities, and non-bankable prices. Under several different scenarios regarding the correlation of shocks across time and regarding the ability of the planner to observe those shocks, I provide expressions (analogous to the well-known result from (Weitzman (1974)) for the expected welfare difference between one policy and another.

This study is the first to my knowledge that models bankable prices. It follows closely from the models in Pizer and Prest (2016) and Weitzman (2018), both of which compare nonbankable prices, non-bankable quantities, and bankable quantities, but omit bankable prices. Like Pizer and Prest (2016), this model allows for policy updating: the planner can observe the first-period shocks before the second period and adjust its policy in response. Like Weitzman (2018), this model allows for shock values that differ across time but are correlated. By allowing for both of these features, this models resolves the somewhat contradictory results from those two papers, in addition to its contribution of modeling bankable prices.

I find that the advantage of bankable prices depends on whether the planner can observe the shocks and whether the shocks differ across periods. In the simplest scenario where the shocks are identical across periods and the planner never observes them, bankability offers no 
advantage to either a price or a quantity policy. When the planner observes the shocks after the first period so policy updating is possible, bankability offers an advantage (in fact, it allows the planner to achieve the first best). But, the advantage is identical for either a bankable price or a bankable quantity policy; and thus bankability does not uniquely confer an advantage upon quantity instruments. When the shocks differ across the two periods and are correlated, but the planner never observes them, then bankable prices does not offer any advantage over nonbankable prices. Finally, in the most complete scenario when the shocks differ across periods and the planner can update policy after observing the first-period shocks, the advantage of bankability is more complicated and cannot a priori be signed. Whenever prices dominate quantities, then bankability dominates non-bankability. However, when quantities dominate prices, then bankability may or may not dominate non-bankability. The comparison between a bankable quantity policy and a non-bankable quantity policy identifies one term in which nonbankable quantities dominate if marginal costs are steeper than marginal benefits, as identified in Weitzman (2018). But in addition to this term, there is a term that represents an unambiguous advantage of bankability, regardless of the slope of marginal cost or benefit curves. This represents the fact that the firm can smooth its production over time in the face of the shock value that it observes.

Lastly, I provide a back-of-the-envelope numerical simulation exercise to gauge the magnitude of the efficiency differences across policies when applied to global climate change policy. Under the base-case parameter values, the bankable price policy strictly dominates all other policies, and the efficiency gain of moving from a non-bankable price policy to a bankable price policy is about one-tenth of the efficiency gain from moving from a quantity policy to a 
price policy. Under other parameterizations, the efficiency gain of bankability can exceed the efficiency gain of a price policy over a quantity policy.

The following section presents the model and results from these four different scenarios regarding the observation of shock values. Then, section III briefly explores the implications through numerical simulations.

\section{Model}

I begin with a simple two-period model to provide the main intuition. Consider the standard specification of quadratic benefits and costs of some choice variable $q_{t}$ as in Weitzman (1974, 2018):

$$
\begin{aligned}
& C\left(q_{t}, \theta_{t}\right)=c_{0}+\left(c_{1}+\theta_{t}\right)\left(q_{t}-\hat{q}\right)+\frac{c_{2}}{2}\left(q_{t}-\hat{q}\right)^{2} \\
& B\left(q_{t}, \eta_{t}\right)=b_{0}+\left(b_{1}+\eta_{t}\right)\left(q_{t}-\hat{q}\right)-\frac{b_{2}}{2}\left(q_{t}-\hat{q}\right)^{2}
\end{aligned}
$$

The random variables, $\theta_{t}$ and $\eta_{t}$, affect the first but not the second derivative of the cost and benefit functions, respectively. That is, they shift the level but not the slope of the marginal cost and marginal benefit functions. In expectation the random variables are equal to zero and uncorrelated, and define $c_{1}=b_{1} \cdot{ }^{1}$ These assumptions imply that in expectation net benefits $B-$ $C$ are maximized at $q_{t}=\hat{q}$. There are two time periods, $t=1$ and $t=2$, and I ignore discounting (i.e. set the discount factor $=1$ ).

I consider four different policies available to the planner, who always seeks to maximize expected welfare (i.e. expected net benefits) $E\left[B\left(q_{1}, \eta_{1}\right)-C\left(q_{1}, \theta_{1}\right)+B\left(q_{2}, \eta_{2}\right)-C\left(q_{2}, \theta_{2}\right)\right]$. First, the planner can set a non-bankable quantity policy $\left\{\tilde{q}_{1}, \tilde{q}_{2}\right\}$, detailing how much the firm

\footnotetext{
${ }^{1}$ This is equivalent to a normalization that ensures that in expectation $\hat{q}$ is the optimal quantity; see Weitzman (2018).
} 
can produce each period. Given this policy, the firm's decision is trivial: it must produce $q_{t}=\tilde{q}_{t}$ in each period.

Second, the planner can set a non-bankable price policy $\left\{\tilde{p}_{1}, \tilde{p}_{2}\right\}$, in which the firm faces a price per unit of output in each period. The firm's optimization problem in period $t$ is

$$
\max _{q_{t}} \tilde{p}_{t} q_{t}-C\left(q_{t}, \theta_{t}\right)
$$

Third, the planner can set a bankable quantity policy, setting $\left\{\tilde{q}_{1}, \tilde{q}_{2}\right\}$ but allowing the firm to choose a quantity $B$ to bank $(B>0)$ or borrow $(B<0)$ between the two periods. ${ }^{2}$ At the start of period 2, the firm would find itself with a bank $B$ and be required to produce $q_{2}=\tilde{q}_{2}+$ $B$. If $B>0$, some of the first period's allotment $\tilde{q}_{1}$ was banked, so the firm can (and must) produce more than its allotment $\tilde{q}_{2}$. If $B<0$, then some of the second period's allotment was borrowed back in the first period, so the firm in the second period must produce less than its allotment $\tilde{q}_{2}$. At the start of period 1 , the firm chooses both its actual quantity produced $q_{1}$ and the amount that it banks or borrows $B$ subject to $q_{1}=\tilde{q}_{1}-B$.

Fourth and finally, the planner can set a bankable price policy, setting $\left\{\tilde{p}_{1}, \tilde{p}_{2}\right\}$ but allowing the firm to choose a quantity $B$ to bank or borrow between periods. If $B>0$, then the firm banks some of its output to the second period, and therefore faces the second-period price on that quantity. Thus, its first-period maximand is $\tilde{p}_{1}\left(q_{1}-B\right)-C\left(q_{1}, \theta_{1}\right)$. In the second period, the firm faces the second-period price on its actual second-period output plus the banked output, so its maximand is $\tilde{p}_{2}\left(q_{2}+B\right)-C\left(q_{2}, \theta_{2}\right)$. When $B<0$, these maximand expressions are unchanged, though the interpretation of $B$ is borrowing from period 2 to period 1.

\footnotetext{
${ }^{2}$ What I refer to as a "bankable" policy is actually both bankable and borrowable; for conciseness I will just use the term "bankable."
} 
For each policy, I solve for the firm's optimal response to a given policy level. Given that, I solve for the planner's optimal policy level, set to maximize expected welfare given the firm's response. Lastly, I solve for expected welfare given optimal policy and optimal firm response. I compare expected welfare across the four policies to see which policy the planner would prefer ex ante. ${ }^{3}$ I do this comparison for several different scenarios involving the specification of the shocks and realization of the uncertainty over the random variables.

\section{II.A. Scenario A: Identical shocks, No policy updating}

I begin with a simple specification of uncertainty before moving on to more interesting and complicated cases. Nonetheless, even in this simplest of scenarios the intuition behind the paper's main results can be seen.

The random variables in this scenario are identical across the two periods; that is, $\theta_{1}=$ $\theta_{2} \equiv \theta$ and $\eta_{1}=\eta_{2} \equiv \eta . E[\theta]=E[\eta]=E[\theta \eta]=0$, and $\sigma_{\theta}^{2} \equiv E\left[\theta^{2}\right]$ and $\sigma_{\eta}^{2} \equiv E\left[\eta^{2}\right]$. The firm observes its random variables before the start of period 1, but the planner never observes them. Therefore, the planner is unable to update the policy between periods 1 and 2 . The firstbest outcome is $q_{1}=q_{2}=\hat{q}+\frac{\eta-\theta}{b_{2}+c_{2}}$. This result and all others are proven in the Appendix.

First consider the non-bankable quantity policy $\left\{\tilde{q}_{1}, \tilde{q}_{2}\right\}$. The firm's response to this policy is trivial: $q_{t}=\tilde{q}_{t}$. Knowing this response, the planner chooses $\tilde{q}_{1}$ and $\tilde{q}_{2}$ to maximize expected net benefits. That is, the planner's problem is

$$
\max _{\tilde{q}_{1}, \tilde{q}_{2}} E\left[B\left(\tilde{q}_{1}, \eta\right)-C\left(\tilde{q}_{1}, \theta\right)+B\left(\tilde{q}_{2}, \eta\right)-C\left(\tilde{q}_{2}, \theta\right)\right]
$$

\footnotetext{
${ }^{3}$ While the planner can choose which of the four policy options yields the highest expected welfare, the firm does not have the option to choose which policy it is subject to, as in Krysiak and Oberauner (2010).
} 
Given the specification of the benefit and cost functions and the structure of the uncertainties, it is straightforward to show that the planner's optimal policy is to set $\tilde{q}_{1}=\tilde{q}_{2}=\hat{q}$. Given this policy and the firm's (trivial) response to it, the expected net benefits of the non-bankable quantity policy is

$$
E W_{N B Q}^{A}=2\left(b_{0}-c_{0}\right)
$$

The acronym $E W$ is for expected welfare (i.e. expected net benefits), the subscript $N B Q$ indicates the non-bankable quantity policy, and the superscript $A$ indicates scenario A (identical shocks, no policy updating).

Second, consider the non-bankable price policy $\left\{\tilde{p}_{1}, \tilde{p}_{2}\right\}$. In response to this policy, the firm chooses the quantity in each period to maximize $\tilde{p}_{t} q_{t}-C\left(q_{t}, \theta\right)$. Each period, this yields the firm's response function $q_{t}=h\left(\tilde{p}_{t}, \theta\right) \equiv \hat{q}+\frac{\tilde{p}_{t}-c_{1}-\theta}{c_{2}}$. Knowing that response, the planner chooses $\tilde{p}_{1}$ and $\tilde{p}_{2}$ to maximize expected net benefits. Solving for the planner's optimal policy yields $\tilde{p}_{1}=\tilde{p}_{2}=c_{1}$ (the planner sets the price equal to the expected marginal cost). Given this optimal policy and the firm's response to it, the expected net benefits of the non-bankable price policy is

$$
E W_{N B P}^{A}=2\left(b_{0}-c_{0}\right)+\frac{\sigma_{\theta}^{2}}{c_{2}^{2}}\left(c_{2}-b_{2}\right)
$$

Comparing this equation to equation 1 , the difference in expected welfare between the nonbankable quantity policy and the non-bankable price policy is

$$
\Delta_{N B P, N B Q}^{A}=\frac{\sigma_{\theta}^{2}}{c_{2}^{2}}\left(c_{2}-b_{2}\right)
$$

Here $\Delta$ indicates the difference in expected welfare between two policies, where the subscript $N B P, N B Q$ indicates that it is the difference between the non-bankable price and the non- 
bankable quantity policy (i.e. $\Delta_{N B P, N B Q}^{A} \equiv E W_{N B P}^{A}-E W_{N B Q}^{A}$ ). The expression for $\Delta_{N B P, N B Q}^{A}$ is simply the standard Weitzman (1974) prices-vs-quantities expression multiplied by two because this is a two-period model.

Third, consider the bankable quantity policy. In period 1 , the firm chooses how much to emit and how much to bank, $B$. If $B<0$, then this means the firm borrows from the next period. For now assume no restrictions on banking or borrowing and no discount or penalty for banking or borrowing. The firm's problem is

$$
\begin{gathered}
\max _{q_{1}, q_{2}, B}-C\left(q_{1}, \theta\right)-C\left(q_{2}, \theta\right) \\
\text { s.t. } q_{1}=\tilde{q}_{1}-B, q_{2}=\tilde{q}_{2}+B
\end{gathered}
$$

Because the firm's cost function is identical across periods (because of the identical shock) and concave, the firm will always want to perfectly smooth out production: $q_{1}=q_{2}=\frac{\tilde{q}_{1}+\tilde{q}_{2}}{2}$. The planner's optimal policy is the same as under the non-bankable quantity policy: $\tilde{q}_{t}=\hat{q}$ in each period (actually the planner can set any pair $\tilde{q}_{1}, \tilde{q}_{2}$ such that $\tilde{q}_{1}+\tilde{q}_{2}=2 \hat{q}$ knowing that the firm will smooth production across periods). As a result, the outcome under this policy will be identical to the outcome under the non-bankable quantity policy from equation $1: E W_{B Q}^{A}=$ $E W_{N B Q}^{A}$ and $\Delta_{N B Q, B Q}^{A}=0$. Under this simplest of assumptions about identical shocks and no policy updating, banking offers no advantage to a quantity policy.

Fourth and finally, consider the bankable price policy. The firm faces prices in each period $\tilde{p}_{1}$ and $\tilde{p}_{2}$. It is able to bank some of its output (or emissions) $B$ and pay the price in the following period. Alternatively, if $B<0$, it borrows forward some of its output from the second period and pays for it at the first-period price. The firm's problem thus is

$$
\max _{q_{1}, q_{2}, B} \tilde{p}_{1}\left(q_{1}-B\right)-C\left(q_{1}, \theta\right)+\tilde{p}_{2}\left(q_{2}+B\right)-C\left(q_{2}, \theta\right)
$$




$$
\text { s.t. } q_{1}-B \geq 0, q_{2}+B \geq 0
$$

The non-negativity constraints ensure that the firm cannot borrow or bank more than the total produced. Other than that, there are no restrictions on banking or borrowing. Given an arbitrary policy $\tilde{p}_{1}, \tilde{p}_{2}$, it can be shown that the firm's problem has the following solution:

$$
q_{1}=q_{2}=\hat{q}+\frac{\max \left\{\tilde{p}_{1}, \tilde{p}_{2}\right\}-c_{1}-\theta}{c_{2}}
$$

For any price pair, the firm chooses to face the price in the higher-price period (if output represents emissions and the price is an emissions tax, then higher price means a lower tax). If period 1 has the higher price, then the firm will borrow all of its quantity from the second period and pay it all in the first period. This is merely the simple intuition that, when the firm can choose its price, it will choose the highest price that it can get. ${ }^{4}$ But, because the cost function is identical across periods and convex, the firm chooses to actually produce an equal amount in each period $\left(q_{1}=q_{2}\right)$. Given the firm's solution, the planner's optimal policy is to set price equal to expected marginal cost in each period, $\tilde{p}_{t}=c_{1}$ (more generally, the planner could set either period's price anything lower than $c_{1}$ and the other period's price equal to $c_{1}$, since the firm will choose to get paid the higher price). It follows that the outcome will be the same under this policy as it is under the non-bankable price policy (equation 2), so that $E W_{B P}^{A}=E W_{N B P}^{A}$ and $\Delta_{N B P, B P}^{A}=0$. Just like with the quantity policy, in scenario A banking offers no advantage to a price policy.

\footnotetext{
${ }^{4}$ In all scenarios considered in this paper, this simple intuition remains, since the firm can always perfectly anticipate the planner's policy and both period's prices. An extension to this framework would be to consider a case where the firm does not know the prices with certainty and may hedge by banking only some of its emissions.
} 
The first row of Table 1 summarizes the expected welfare under each of the four policy options, each expressed relative to expected welfare under non-bankable quantities $\left(2\left(b_{0}-c_{0}\right)\right)$. The remaining rows present the corresponding expressions for the remaining scenarios below.

\section{B. Scenario B: Identical shocks, Policy updating}

Maintain the assumption that the shocks are identical across periods. But now the planner is able to observe the value of the shocks $\theta$ and $\eta$ at the end of period 1 and update its period 2 policy in response..$^{5}$ This is identical to the main specification of uncertainty in Pizer and Prest (2016). As in scenario A, the ex-post first-best output level is $q_{1}=q_{2}=\hat{q}+\frac{\eta-\theta}{b_{2}+c_{2}}$.

Under a non-bankable price policy the firm's problem is again trivial $\left(q_{t}=\tilde{q}_{t}\right)$. The planner's problem can be considered separately by the two periods, since the planner has different information in each period. At the start of period 2, the planner observes the values of the shocks and chooses its quantity constraint $\tilde{q}_{2}$. The planner's optimal policy in period 2 is to set the constraint just equal to the optimal quantity: $\tilde{q}_{2}=\hat{q}+\frac{\eta-\theta}{b_{2}+c_{2}}$. In the first period, the planner knows that it will set that quantity in the second period but doesn't yet know what values the shocks take. Choosing the first-period quantity that maximizes expected welfare yields $\tilde{q}_{1}=$ $\hat{q}$. Given this policy, the expected welfare of the non-bankable price policy is

$$
E W_{N B Q}^{B}=2\left(b_{0}-c_{0}\right)+\frac{1}{2}\left(\frac{\sigma_{\eta}^{2}+\sigma_{\theta}^{2}}{b_{2}+c_{2}}\right)
$$

\footnotetext{
${ }^{5}$ The planner can update in response to the observed values of the shocks but not in response to observing the level of the bank $B$ as in Kollenberg and Taschini (2016).
} 
For a non-bankable price policy, the firm's response to policy is identical to what it is in scenario A: $q_{t}=h\left(\tilde{p}_{t}, \theta\right) \equiv \hat{q}+\frac{\tilde{p}_{t}-c_{1}-\theta}{c_{2}}$. At the start of period 2, the planner observes the shocks and sets the price policy to maximize welfare. This results in the planner achieving the first-best output level in period 2, $q_{2}=\hat{q}+\frac{\eta-\theta}{b_{2}+c_{2}}$, by setting $\tilde{p}_{2}=c_{1}+\frac{b_{2} \theta+c_{2} \eta}{b_{2}+c_{2}}$. In the first period, the planner will set $\tilde{p}_{1}=c_{1}$ (without observing the shock value the planner just sets price equal to expected marginal cost), which yields $q_{1}=\hat{q}-\frac{\theta}{c_{2}}$. As a result, the expected welfare will be

$$
E W_{N B P}^{B}=2\left(b_{0}-c_{0}\right)+\frac{1}{2}\left(\frac{\sigma_{\eta}^{2}+\sigma_{\theta}^{2}}{b_{2}+c_{2}}\right)+\frac{\sigma_{\theta}^{2}}{2 c_{2}^{2}}\left(c_{2}-b_{2}\right)
$$

Comparing this equation to equation 5 yields

$$
\Delta_{N B P, N B Q}^{B}=\frac{\sigma_{\theta}^{2}}{2 c_{2}^{2}}\left(c_{2}-b_{2}\right)
$$

This expression is identical to the original Weitzman (1974) prices vs. quantities expression, because here the two outcomes are identical to each other in the second period, and in the first period the problem is identical to the one-period Weitzman (1974) problem.

Next is the bankable quantity policy. Just as in scenario A without policy updating, the firm will always want to perfectly smooth its output across the two periods, given its identical and convex cost curves and its unlimited ability to bank and borrow. So, for any allocated quantities $\tilde{q}_{1}$ and $\tilde{q}_{2}$ it will choose a level of banking or borrowing to achieve $q_{1}=q_{2}=\frac{\tilde{q}_{1}+\tilde{q}_{2}}{2}$. In the second period, after the planner has observed the values of the shocks, it will choose a second-period policy level $\tilde{q}_{2}$ to induce the optimal level of second-period output $q_{2}=\hat{q}+$ 
$\frac{\eta-\theta}{b_{2}+c_{2}}$. To induce this, the planner can set any arbitrary quantity $\tilde{q}_{1}$ in the first period, knowing that once it observes the shocks before the second period, it can respond by setting its secondperiod quantity policy so that the sum $\tilde{q}_{1}+\tilde{q}_{2}=2\left(\hat{q}+\frac{\eta-\theta}{b_{2}+c_{2}}\right)$, in which case the firm will smooth and achieve the optimal output in each period. ${ }^{6}$ Therefore, a bankable quantity policy with updating yields the first-best, as shown in Pizer and Prest (2016). This first-best outcome results in expected welfare of

$$
E W_{B Q}^{B}=2\left(b_{0}-c_{0}\right)+\left(\frac{\sigma_{\eta}^{2}+\sigma_{\theta}^{2}}{b_{2}+c_{2}}\right)
$$

Comparing this to equation 5 yields the difference

$$
\Delta_{B Q, N B Q}^{B}=\frac{1}{2}\left(\frac{\sigma_{\eta}^{2}+\sigma_{\theta}^{2}}{b_{2}+c_{2}}\right)>0
$$

Bankable quantities dominate non-bankable quantities. Furthermore, comparing bankable quantities to non-bankable prices (equations 8 and 6) yields

$$
\Delta_{B Q, N B P}^{B}=\frac{1}{2\left(b_{2}+c_{2}\right)}\left[\sigma_{\eta}^{2}+\left(\frac{b_{2}}{c_{2}}\right)^{2} \sigma_{\theta}^{2}\right]>0
$$

This is the main result from Pizer and Prest (2016): bankable quantities dominate non-bankable prices under policy updating.

However, Pizer and Prest (2016) do not allow for bankable prices. The firm's response to this policy is the same as its response under scenario A:

$$
q_{1}=q_{2}=\hat{q}+\frac{\max \left\{\tilde{p}_{1}, \tilde{p}_{2}\right\}-c_{1}-\theta}{c_{2}}
$$

\footnotetext{
${ }^{6}$ The firm observes its shocks before period 1, and the firm knows what the planner's response to any arbitrary shock will be, therefore the firm can perfectly anticipate the planner's second-period strategy at the start of the first period though the planner does not choose it until the second period.
} 
That is, just as with bankable prices, the firm will perfectly smooth its output over the two periods. Furthermore, the firm will be able to "choose" its price among the two period's prices by banking or borrowing such that it receives the maximum of the two prices. Given this firm response, the planner is able to induce the first best in both periods. In the second period, after observing the shock, it sets the price that induces the optimal outcome, $\tilde{p}_{2}=c_{1}+\frac{b_{2} \theta+c_{2} \eta}{b_{2}+c_{2}}$. The first-period price can be anything arbitrary low enough to ensure that it is always lower than the first-period price. Thus, the planner knows that the firm will actually face the second-period price, and it knows that by the second period it will have enough information to set that price to achieve the first-best. Therefore, the bankable price policy induces the first-best outcome $q_{1}=$ $q_{2}=\hat{q}+\frac{\eta-\theta}{b_{2}+c_{2}}$, just as the bankable quantity policy does, so that $\Delta_{B Q, B P}^{B}=0$. In contrast to the result from Pizer and Prest (2016), here quantities do not strictly dominate prices under policy updating. They do so in the Pizer and Prest (2016) model where only quantities are allowed to be banked. But, when a price policy also allows banking, then both the price and the quantity policy are equivalent (and both strictly dominate a non-bankable price policy and a non-bankable quantity policy).

The specification of uncertainty that allows for policy updating (used here in Scenario B and also in Scenario D) requires some assumptions that may be seen as heroic. The firm and planner are engaged in a mutual rational expectations equilibrium where each one's optimal action is contingent on a self-fulfilling expectation of the other's action. The firm knows its shock values from the start, and it knows that the planner will observe those values only after period 1. The firm knows that the planner will update optimally after period 1 and makes its decisions with that in mind. The planner anticipates the firm's decision and sets its policy with 
that in mind. This degree of rationality may be unrealistic to assume in a situation where realworld regulators are interacting with real-world firms.

The second row of Table 1 summarizes the results for Scenario B. As in the first row, each expected welfare expression is relative to the expected welfare of non-bankable quantities in scenario $\mathrm{A}$; that is, it omits the term $2\left(b_{0}-c_{0}\right)$.

\section{C. Scenario C: Correlated shocks, No policy updating}

In this scenario no longer allow for policy updating; that is, the planner never observes the shocks and so cannot alter the policy between the two periods. However, here the shocks are not identical across the two periods. Instead, they follow autoregressive processes given by:

$$
\begin{aligned}
& \theta_{2}=\rho_{\theta} \theta_{1}+\varepsilon_{\theta} \\
& \eta_{2}=\rho_{\eta} \eta_{1}+\varepsilon_{\eta}
\end{aligned}
$$

The first-period shock values $\theta_{1}$ and $\eta_{1}$ are normally distributed with variances $\sigma_{\theta}^{2}$ and $\sigma_{\eta}^{2}$, respectively, and they are independent of the second-period innovations $\varepsilon_{\theta}$ and $\varepsilon_{\eta}$. Thus, for the planner, as in the previous scenarios, $E\left[\theta_{1}\right]=E\left[\theta_{2}\right]=E\left[\eta_{1}\right]=E\left[\eta_{2}\right]=E\left[\theta_{l} \eta_{m}\right]=0$, and $E\left[\theta_{1}^{2}\right]=\sigma_{\theta}^{2}, E\left[\eta_{1}^{2}\right]=\sigma_{\eta}^{2}$. However, now $E\left[\theta_{2}^{2}\right]=\sigma_{\theta}^{2}\left(\rho_{\theta}^{2}+1\right), E\left[\theta_{1} \theta_{2}\right]=\rho_{\theta} \sigma_{\theta}^{2}, E\left[\eta_{2}^{2}\right]=$ $\sigma_{\eta}^{2}\left(\rho_{\eta}^{2}+1\right), E\left[\eta_{1} \eta_{2}\right]=\rho_{\eta} \sigma_{\eta}^{2} \cdot{ }^{7}$ In this scenario the ex-post first-best outcome is $q_{1}=\hat{q}+\frac{\eta_{1}-\theta_{1}}{b_{2}+c_{2}}$ and $q_{2}=\hat{q}+\frac{\eta_{2}-\theta_{2}}{b_{2}+c_{2}}$

\footnotetext{
${ }^{7}$ Weitzman (2018), which allows for correlated heterogeneous shocks but not policy updating, considers a more general specification that allows for any joint distribution of the two periods' shock values. The specification here is a special case of that more general specification, although the intuitions gleaned from the general case can also be gleaned from the special case.
} 
First consider the non-bankable quantity policy. The firm's response to this policy is as always trivial: $q_{1}=\tilde{q}_{1} ; q_{2}=\tilde{q}_{2}$. Given this response, the planner chooses $\tilde{q}_{1}=\tilde{q}_{2}=\hat{q}$ to maximize expected welfare, which ends up being

$$
E W_{N B Q}^{C}=2\left(b_{0}-c_{0}\right)
$$

Next consider the non-bankable price policy. The firm's response to these prices is the same as in the previous scenarios, except now noting that the firm observes different shocks each period: $q_{1}=\hat{q}+\frac{\tilde{p}_{1}-c_{1}-\theta_{1}}{c_{2}} ; q_{2}=\hat{q}+\frac{\tilde{p}_{2}-c_{1}-\theta_{2}}{c_{2}}$. Knowing these responses but only knowing the distribution and not the values of all of the shocks, the planner maximizes expected welfare by choosing $\tilde{p}_{1}=\tilde{p}_{2}=c_{1}$; that is, the planner sets price equal to expected marginal damages (just as in scenario A). Then, given this optimal policy, the expected welfare under the non-bankable price policy is

$$
E W_{N B P}^{C}=2\left(b_{0}-c_{0}\right)+\frac{\sigma_{\theta}^{2}}{2 c_{2}^{2}}\left(\rho_{\theta}^{2}+2\right)\left(c_{2}-b_{2}\right)
$$

It follows that the advantage of non-bankable prices over non-bankable quantities is

$$
\Delta_{N B P, N B Q}^{C}=\frac{\sigma_{\theta}^{2}}{c_{2}}\left(\rho_{\theta}^{2}+2\right)\left(c_{2}-b_{2}\right)
$$

This is a slightly modified version of the standard Weitzman (1974) result, accounting for the two periods and the correlation of the cost shock across periods $\rho_{\theta}$. This result is identical to the corresponding result in Weitzman (2018) (his equation 42) after imposing the specification of the AR process for the shock.

Next consider the bankable quantity policy. Now, the firm will still want to smooth output over the two periods since it is free to bank and borrow. However, it will not perfectly 
smooth to the point where $q_{1}=q_{2}$, since the shocks are different across periods. Its costminimizing response to the policy is

$$
\begin{aligned}
& q_{1}=\frac{1}{2}\left(\tilde{q}_{1}+\tilde{q}_{2}\right)-\frac{\theta_{1}-\theta_{2}}{2 c_{2}} \\
& q_{2}=\frac{1}{2}\left(\tilde{q}_{1}+\tilde{q}_{2}\right)+\frac{\theta_{1}-\theta_{2}}{2 c_{2}} \\
& B=\frac{1}{2}\left(\tilde{q}_{1}-\tilde{q}_{2}\right)+\frac{\theta_{1}-\theta_{2}}{2 c_{2}}
\end{aligned}
$$

The smoothness is perfect across the two periods $\left(\frac{1}{2}\left(\tilde{q}_{1}+\tilde{q}_{2}\right)\right)$ except for the adjustment $\frac{\theta_{1}-\theta_{2}}{2 c_{2}}$. If that expression is positive, i.e. $\theta_{1}>\theta_{2}$, then the cost is higher in the first period than it is in the second period, so the firm produces a little bit less in the first period than in the second period and vice versa. Only the bank value $B$ depends on the difference in the assigned quantities across periods; the chosen output levels only depend on their sum and on the shocks. Given this firm response, the planner can set the quantities $\tilde{q}_{1}$ and $\tilde{q}_{2}$ to maximize expected welfare. However, as in the previous scenarios, the planner actually need only choose the sum $\tilde{q}_{1}+\tilde{q}_{2}$, since firms will respond just to that sum. ${ }^{8}$ The optimal policy for the planner is $\tilde{q}_{1}+$ $\tilde{q}_{2}=2 \hat{q}$; i.e., it chooses the expected value of welfare-maximizing quantity. Lastly, given this optimal policy by the firm, the expected welfare under this policy is

$$
E W_{B Q}^{C}=2\left(b_{0}-c_{0}\right)+\frac{\sigma_{\theta}^{2}}{4 c_{2}^{2}}\left(\rho_{\theta}^{2}-2 \rho_{\theta}+2\right)\left(c_{2}-b_{2}\right)
$$

(This is identical to the corresponding equation in Weitzman (2018), equation 25, after imposing the AR structure.) The term in parenthesis $\left(\rho_{\theta}^{2}-2 \rho_{\theta}+2\right)$ is strictly positive since $0<\rho_{\theta}<1$.

\footnotetext{
${ }^{8}$ That is, the firm's response to $\left\{\tilde{q}_{1}, \tilde{q}_{2}\right\}$ is identical to its response to $\left\{\tilde{q}_{1}+x, \tilde{q}_{2}-x\right\}$.
} 
At this point, before considering the bankable price policy, compare the three policies considered so far in scenario C. These are the same three policies considered in Weitzman (2018), which (like Pizer and Prest (2016)) ignores bankable prices. As shown earlier, the advantage of non-bankable prices over non-bankable quantities is $\Delta_{N B P, N B Q}^{C}=\frac{\sigma_{\theta}^{2}}{c_{2}}\left(\rho_{\theta}^{2}+2\right)\left(c_{2}-\right.$ $b_{2}$ ), which is positive whenever $c_{2}>b_{2}$, i.e. marginal cost is steeper than marginal benefit. The advantage of bankable quantities over non-bankable quantities is

$$
\Delta_{B Q, N B Q}^{C}=\frac{\sigma_{\theta}^{2}}{4 c_{2}^{2}}\left(\rho_{\theta}^{2}-2 \rho_{\theta}+2\right)\left(c_{2}-b_{2}\right)
$$

This expression has the same sign as $\left(c_{2}-b_{2}\right)$, so bankable quantities dominate non-bankable quantities if and only if marginal cost is steeper than marginal benefit. This is identical to the surprising result in Weitzman (2018) (his equation 47) that bankable quantities are not unambiguously preferred to non-bankable quantities. The explanation is that bankable quantities are unambiguously preferred on cost grounds, but not necessarily so when benefits are factored in as well (as they are in the calculation of expected welfare). The non-bankable quantity policy keeps quantity equal across periods, which is definitely not cost-effective. But, maintaining a constant quantity is welfare-improving vis-à-vis benefits. When marginal benefits are steeper than marginal costs, this smoothing over benefits dominates the lack of smoothing over costs. The advantage of bankable quantities over non-bankable prices is

$$
\Delta_{B Q, N B P}^{C}=\frac{\sigma_{\theta}^{2}}{4 c_{2}^{2}}\left(\rho_{\theta}^{2}+2 \rho_{\theta}+2\right)\left(b_{2}-c_{2}\right)
$$

This is positive whenever $b_{2}>c_{2}$, so bankable quantities dominate non-bankable prices if and only if marginal benefit is steeper than marginal cost. When comparing only these three policies, this yields the result that non-bankable prices dominate bankable quantities, which dominate 
non-bankable quantities whenever $c_{2}>b_{2}$, and that non-bankable quantities dominate bankable quantities, which dominate non-bankable prices, whenever $c_{2}>b_{2}$, the result found in Weitzman (2018).

Does adding the fourth policy option - bankable prices - affect that ranking? The answer is no, because bankable prices in this scenario are equivalent to non-bankable prices. To see that, consider first the firm's response under bankable prices. It will still choose the period with the highest price to bank or borrow all of its quantity to, and optimize in each period based on that price. Its optimal output in each period is

$$
\begin{aligned}
& q_{1}=\hat{q}+\frac{\max \left\{\tilde{p}_{1}, \tilde{p}_{2}\right\}-c_{1}-\theta_{1}}{c_{2}} \\
& q_{2}=\hat{q}+\frac{\max \left\{\tilde{p}_{1}, \tilde{p}_{2}\right\}-c_{1}-\theta_{2}}{c_{2}}
\end{aligned}
$$

The planner thus only needs to choose one effective price $\tilde{p}$, and its optimal price is $\tilde{p}=c_{1}$. In response, the firm will choose $q_{1}=\hat{q}-\frac{\theta_{1}}{c_{2}}$ and $q_{2}=\hat{q}-\frac{\theta_{2}}{c_{2}}$, and this will result in expected welfare of

$$
E W_{B P}^{C}=2\left(b_{0}-c_{0}\right)+\frac{\sigma_{\theta}^{2}}{2 c_{2}^{2}}\left(\rho_{\theta}^{2}+2\right)\left(c_{2}-b_{2}\right)
$$

This expression is identical to the expression for $E W_{N B P}^{C}$ in equation 13, since under either policy the planner chooses price such that the firm faces the expected marginal $\operatorname{cost} c_{1}$ in each period. It follows that in scenario $\mathrm{C}$, the addition of bankable prices does not change the ranking found earlier when just considering the three other policies. Unlike in the case with policy updating (scenario B), here in scenario $\mathrm{C}$ with correlated shocks, bankability provides no advantage over non-bankability to a price policy. 
The third row of Table 1 summarizes the results from scenario C. It is not the case that scenario $\mathrm{C}$ reduces to scenario A when $\rho_{\theta}=\rho_{\eta}=1$, because there are innovations in the second-period ( $\varepsilon_{\theta}$ and $\left.\varepsilon_{\eta}\right)$ that prevent the two periods' shock values from being identical to each other. ${ }^{9}$ Furthermore, when $\rho_{\theta}=0$, expected welfare under scenarios A and $\mathrm{C}$ are identical to each other for both price policies, though it is not the case that the two scenarios are identical to each other.

\section{D. Scenario D: Correlated shocks, policy updating}

The final scenario is the most general, since it includes both correlated shocks and policy updating. After the first period, the planner observes the values of the first-period shocks $\theta_{1}$ and $\eta_{1}$ and can set the second-period policy in response to it. The planner never observes the secondperiod shocks. This scenario thus generalizes both the model from Pizer and Prest (2016), which considers only policy updating, and from Weitzman (2018), which considers only correlated shocks. In addition to this generalization of those two models, here I also consider a bankable price policy in addition to the other three policies. As in scenario $\mathrm{C}$, the ex-post first-best outcome is $q_{1}=\hat{q}+\frac{\eta_{1}-\theta_{1}}{b_{2}+c_{2}}$ and $q_{2}=\hat{q}+\frac{\eta_{2}-\theta_{2}}{b_{2}+c_{2}}$.

First I consider the non-bankable quantity policy, where the firm's problem is again trivial since it must choose $q_{1}=\tilde{q}_{1}$ and $q_{2}=\tilde{q}_{2}$. The planner will set

$$
\begin{gathered}
q_{2}=\hat{q}+\frac{\rho_{\eta} \eta_{1}-\rho_{\theta} \theta_{1}}{b_{2}+c_{2}} \\
q_{1}=\hat{q}
\end{gathered}
$$

\footnotetext{
${ }^{9}$ In order to nest scenario A as a special case of scenario $\mathrm{C}$, I would have to specify that the innovation to the second-period cost shock $\left(\varepsilon_{\theta}\right)$ has a different variance (say, $\sigma_{\theta 2}^{2}$ ) than the first-period cost shock (say, $\sigma_{\theta 1}^{2}$ ), and likewise for the benefit shocks. This would introduce another variable and in my assessment make the model less tractable without providing additional insight.
} 
In both periods, the planner sets the quantity policy equal to the value that maximizes expected net welfare, conditional on the planner's information set. In period 1, before observing any shocks, that value is just $\hat{q}$. After the planner observes the first-period shocks, the optimal quantity is updated. Given this optimal policy, the expected welfare is

$$
E W_{N B Q}^{D}=2\left(b_{0}-c_{0}\right)+\frac{1}{2} \frac{\rho_{\eta}^{2} \sigma_{\eta}^{2}+\rho_{\theta}^{2} \sigma_{\theta}^{2}}{b_{2}+c_{2}}
$$

Next consider the non-bankable price policy. As in scenario B, the firm's response to the pair of prices is to produce $q_{1}=\hat{q}+\frac{\tilde{p}_{1}-c_{1}-\theta_{1}}{c_{2}}$ and $q_{2}=\hat{q}+\frac{\tilde{p}_{2}-c_{1}-\theta_{2}}{c_{2}}$. The planner's problem is again in two stages: the period 2 policy $\tilde{p}_{2}$ is chosen after the observation of the first-period shocks, while the period 1 policy $\tilde{p}_{1}$ is chosen before. The planner's optimal policy is

$$
\begin{gathered}
\tilde{p}_{1}=c_{1} \\
\tilde{p}_{2}=c_{1}+\frac{c_{2} \rho_{\eta} \eta_{1}+b_{2} \rho_{\theta} \theta_{1}}{b_{2}+c_{2}}
\end{gathered}
$$

This pair of prices will result in the firm producing $q_{1}=\hat{q}-\frac{\theta_{1}}{c_{2}}$ and $q_{2}=\hat{q}+\frac{1}{c_{2}} \frac{c_{2} \rho_{\eta} \eta_{1}+b_{2} \rho_{\theta} \theta_{1}}{b_{2}+c_{2}}-$ $\frac{\theta_{2}}{c_{2}}$. Substituting these values into the expression for expected welfare (evaluated before observing the values of the shocks) yields

$$
E W_{N B P}^{D}=2\left(b_{0}-c_{0}\right)+\frac{1}{2} \frac{\rho_{\eta}^{2} \sigma_{\eta}^{2}+\rho_{\theta}^{2} \sigma_{\theta}^{2}}{b_{2}+c_{2}}+\frac{\sigma_{\theta}^{2}}{c_{2}^{2}}\left(c_{2}-b_{2}\right)
$$

Comparing expected welfare under the non-bankable quantity policy to expected welfare under the non-bankable price policy yields the difference

$$
\Delta_{N B P, N B Q}^{D}=\frac{\sigma_{\theta}^{2}}{c_{2}^{2}}\left(c_{2}-b_{2}\right)
$$


Prices dominate quantities whenever $c_{2}>b_{2}$, as in Weitzman (1974).

Next consider the bankable quantity policy. Just as in scenario C, the firm's response to policy is $q_{1}=\frac{1}{2}\left(\tilde{q}_{1}+\tilde{q}_{2}\right)-\frac{\theta_{1}-\theta_{2}}{2 c_{2}}$ and $q_{2}=\frac{1}{2}\left(\tilde{q}_{1}+\tilde{q}_{2}\right)+\frac{\theta_{1}-\theta_{2}}{2 c_{2}}$. Here the planner's policy choice is to set the sum $\tilde{q}_{1}+\tilde{q}_{2}$ to maximize expected welfare. After the first period the planner observes $\theta_{1}$ and $\eta_{1}$, and so it can set its second-period policy $\tilde{q}_{2}$ as a function of these variables. It cannot do so in the first period. However, the planner can choose any arbitrarily low firstperiod policy (e.g. $\tilde{q}_{1}=0$ ) knowing that the firm will respond only to the sum of the two policies $\tilde{q}_{1}+\tilde{q}_{2}$, and so the firm can set this sum after the second period and in response to the firstperiod shock values. The planner's problem is thus to choose the sum $\tilde{q}_{1}+\tilde{q}_{2}$ to maximize expected welfare, conditional on observing the first-period shocks, and subject to constraints given by the firm's response. This problem yield's the planner's solution $\tilde{q} \equiv \frac{1}{2}\left(\tilde{q}_{1}+\tilde{q}_{2}\right)=\hat{q}+$ $\frac{1}{2} \frac{\eta_{1}\left(1+\rho_{\eta}\right)-\theta_{1}\left(1+\rho_{\theta}\right)}{b_{2}+c_{2}}$. The planner sets the average quantity across the two periods equal to the planner's best guess about what the optimal quantity will be on average, knowing only the firstperiod shocks. The firm's response to this policy is

$$
\begin{aligned}
& q_{1}=\hat{q}+\frac{1}{2} \frac{\eta_{1}\left(1+\rho_{\eta}\right)-\theta_{1}\left(1+\rho_{\theta}\right)}{b_{2}+c_{2}}-\frac{\theta_{1}-\theta_{2}}{2 c_{2}} \\
& q_{2}=\hat{q}+\frac{1}{2} \frac{\eta_{1}\left(1+\rho_{\eta}\right)-\theta_{1}\left(1+\rho_{\theta}\right)}{b_{2}+c_{2}}+\frac{\theta_{1}-\theta_{2}}{2 c_{2}}
\end{aligned}
$$

Evaluating ex-ante expected welfare (not conditional on the first-period shocks), given these quantity values, yields

$$
\begin{aligned}
E W_{B Q}^{D}=2\left(b_{0}\right. & \left.-c_{0}\right)+\frac{1}{4} \frac{\left(1+\rho_{\eta}\right)^{2} \sigma_{\eta}^{2}+\left(1+\rho_{\theta}\right)^{2} \sigma_{\theta}^{2}}{b_{2}+c_{2}} \\
+ & \frac{\sigma_{\theta}^{2}}{4 c_{2}^{2}}\left(c_{2}-b_{2}\right)\left(2+\rho_{\theta}^{2}-2 \rho_{\theta}\right)
\end{aligned}
$$


Comparing this expected welfare to that of the expected welfare under non-bankable prices yields the difference

$$
\begin{aligned}
& \Delta_{B Q, N B Q}^{D}=\frac{\sigma_{\theta}^{2}}{4 c_{2}^{2}}\left(c_{2}-b_{2}\right)\left(2+\rho_{\theta}^{2}-2 \rho_{\theta}\right) \\
&+\frac{1}{4} \frac{\sigma_{\eta}^{2}\left(\left(1+\rho_{\eta}\right)^{2}-2 \rho_{\eta}^{2}\right)+\sigma_{\theta}^{2}\left(\left(1+\rho_{\theta}\right)^{2}-2 \rho_{\theta}^{2}\right)}{b_{2}+c_{2}}
\end{aligned}
$$

The first term in this expression has the same sign as $c_{2}-b_{2}$. This term is analogous to the result found here in equation 16 and in Weitzman (2018) that bankable quantities dominate nonbankable quantities only when $c_{2}>b_{2}$. However, in addition there is the second term of equation 23 , which is strictly nonnegative. This second term captures the fact that there is an advantage of bankability, regardless of the slopes of the marginal cost and marginal benefit curves, since the firm (which has more information than the planner) is able to smooth out the shocks and reduce costs with bankability. This term is missing in the model from Weitzman (2018) since that model does not consider policy updating.

Lastly consider the bankable price policy. As in scenario C, the firm's optimal response to a policy is $q_{1}=\hat{q}+\frac{\max \left\{\tilde{p}_{1}, \tilde{p}_{2}\right\}-c_{1}-\theta_{1}}{c_{2}} ; q_{2}=\hat{q}+\frac{\max \left\{\tilde{p}_{1}, \tilde{p}_{2}\right\}-c_{1}-\theta_{2}}{c_{2}}$. The planner's problem is similar to its problem under the bankable quantity policy - since it observes the first-period shock values before the start of the second period, it can set its first-period price arbitrarily low so that the second-period price is always binding, and therefore it can set this price to maximize expected welfare conditional on observing the first-period shock values. Doing so, the planner

chooses $\tilde{p}=c_{1}+\frac{1}{2\left(b_{2}+c_{2}\right)}\left(b_{2}\left(1+\rho_{\theta}\right) \theta_{1}+c_{2}\left(1+\rho_{\eta}\right) \eta_{1}\right)$. This is the planner's expected value of the average marginal cost across the two periods given its observation of the first-period shocks. In response to this policy, the firm chooses to produce 


$$
\begin{aligned}
& q_{1}=\hat{q}+\frac{1}{2 c_{2}} \frac{c_{2} \eta_{1}\left(1+\rho_{\eta}\right)+b_{2} \theta_{1}\left(1+\rho_{\theta}\right)}{b_{2}+c_{2}}-\frac{\theta_{1}}{c_{2}} \\
& q_{2}=\hat{q}+\frac{1}{2 c_{2}} \frac{c_{2} \eta_{1}\left(1+\rho_{\eta}\right)+b_{2} \theta_{1}\left(1+\rho_{\theta}\right)}{b_{2}+c_{2}}-\frac{\theta_{2}}{c_{2}}
\end{aligned}
$$

Ex-ante expected welfare given these quantities produced is

$$
\begin{aligned}
E W_{B P}^{D}=2\left(b_{0}\right. & \left.-c_{0}\right)+\frac{1}{4} \frac{\left(1+\rho_{\eta}\right)^{2} \sigma_{\eta}^{2}+\left(1+\rho_{\theta}\right)^{2} \sigma_{\theta}^{2}}{b_{2}+c_{2}} \\
& +\frac{\sigma_{\theta}^{2}}{4 c_{2}^{2}}\left(c_{2}-b_{2}\right)\left(3+\rho_{\theta}^{2}-2 \rho_{\theta}\right)
\end{aligned}
$$

The second term is positive (and is identical to a term in the expression for $E W_{B Q}^{D}$ ), and the third term has the same sign as $c_{2}-b_{2}\left(\right.$ since $\left.3+\rho_{\theta}^{2}-2 \rho_{\theta}>0\right)$. This expression can be compared with both $E W_{N B P}^{D}$, to see to advantage that bankability offers to prices, and with $E W_{B Q}^{D}$, to see the advantage that prices have over quantities when both are bankable. First,

$$
\begin{aligned}
\Delta_{B P, N B P}^{D}=\frac{\sigma_{\theta}^{2}}{4 c_{2}^{2}} & \left(c_{2}-b_{2}\right)\left(-1+\rho_{\theta}^{2}-2 \rho_{\theta}\right) \\
& +\frac{1}{4} \frac{\sigma_{\eta}^{2}\left(\left(1+\rho_{\eta}\right)^{2}-2 \rho_{\eta}^{2}\right)+\sigma_{\theta}^{2}\left(\left(1+\rho_{\theta}\right)^{2}-2 \rho_{\theta}^{2}\right)}{b_{2}+c_{2}}
\end{aligned}
$$

The first term has the opposite sign as $c_{2}-b_{2}$. Compare that to the first term in equation 23, which has the same sign as $c_{2}-b_{2}$. These terms represent the same effect, which goes in opposite directions for bankable prices and bankable quantities. When marginal cost is steeper than marginal benefit, there is an effect that yields an advantage to bankability for quantity policies. This same effect yields an advantage to non-bankability over bankability for quantity when marginal benefit is steeper than marginal cost. This is the effect identified in Weitzman (2018). But for price policies, this effect works in the opposite direction. When marginal cost is 
steeper than marginal benefit, non-bankability is preferred to bankability for price policies, and when marginal benefit is steeper than marginal cost, bankability is preferred.

The explanation for this, just like the explanation for the first term in equation 23 , lies in the trade-off between achieving cost-effectiveness and achieving "benefit-effectiveness," or smoothing between periods so that marginal benefits are equal. For a quantity policy, making it bankable ensures cost-effectiveness since firms will smooth to minimize costs, but this may come at a cost of reducing benefits. For a price policy, making it bankable ensures that the firm faces the same price in each period, which is not necessarily cost-effective since the cost shocks vary across the two periods. But, relative to non-bankable prices, it forces the firm to more efficiently internalize benefits. Thus, when marginal benefits are steeper and internalizing these benefits is relatively more important, bankability dominates non-bankability for prices.

In addition to that effect, there is another effect, captured in the second term in equation 25 , and also in the second term in equation 23 , that is unambiguously positive, so that it yields an advantage to bankability regardless of the relative slopes, for either quantities or prices. This term is absent in Weitzman (2018) and is due to policy updating - the planner's ability to do so allows the firm to more cost-effectively smooth output over the two periods than it otherwise would have.

Equation 25 demonstrates that when $c_{2}>b_{2}$ there are two offsetting effects in the ranking of bankable versus non-bankable prices. The first term is negative and the second term is positive. However, it can be shown that the first term is always dominated by the second term so that the sign of $\Delta_{B P, N B P}^{D}$ is always positive. Equation 25 can be rearranged to yield

$$
\Delta_{B P, N B P}^{D}=\frac{\sigma_{\theta}^{2} b_{2}^{2}}{4 c_{2}^{2}\left(b_{2}+c_{2}\right)}\left(1-\rho_{\theta}^{2}+2 \rho_{\theta}\right)+\frac{1}{4} \frac{\sigma_{\eta}^{2}\left(\left(1+\rho_{\eta}\right)^{2}-2 \rho_{\eta}^{2}\right)}{b_{2}+c_{2}}
$$


All terms in equation 26 are unambiguously positive. Thus there is an important distinction between the advantage that bankability offers to price policies and the advantage that it offers to quantity policies. Bankability is unambiguously preferred for price policies, but sometimes nonbankability can dominate bankability for quantity policies, as shown in equation 23 . Later, in the numerical simulations, I verify this claim by finding parameter values that make it true.

Next,

$$
\Delta_{B P, B Q}^{D}=\frac{\sigma_{\theta}^{2}}{4 c_{2}^{2}}\left(c_{2}-b_{2}\right)
$$

This has the same sign as $c_{2}-b_{2}$. Just as in the original Weitzman (1974) model, prices dominate quantities whenever marginal cost is steeper than marginal benefit; this holds even when both prices and quantities are bankable, and even when shocks are correlated and policy updating is available.

Comparing all four policy options in Scenario D allows me to make the following claim. When $c_{2}>b_{2}$, then non-bankable prices dominate non-bankable quantities (from $\Delta_{N B P, N B Q}^{D}$, the standard Weitzman (1974) result), bankable prices dominate bankable quantities (from $\Delta_{B P, B Q}^{D}$ ), and bankable prices dominate non-bankable prices (from $\Delta_{B P, N B P}^{D}$ in equation 26). When $c_{2}<$ $b_{2}$, then non-bankable quantities dominate non-bankable prices, bankable quantities dominate bankable prices, but the advantage of bankable quantities over non-bankable quantities is ambiguous (from $\Delta_{B Q, N B Q}^{D}$ ). That is, quantities unambiguously dominate prices, but whether bankability dominates non-bankability depends upon the parameter values.

\section{E. Multi-period $(T>2)$}


All of the previous scenarios consider just two time periods, but the basic intuitions derived in them extend to a model with more than two time periods. For instance, when a policy-maker can update after each period, she will choose to set the policy in the period in which she has the most information, which is the final period. With bankable quantities, the quantity allocated in the final period will determine the total quantity available, and with bankable prices, the price set in the final period will be the binding price. In other words, while in the two-period model, the policy-maker will "true up" the policy in the second period after observing the shock, when $T>2$ the policy-maker will true up after observing as much as possible in the final period.

More concretely, consider the extension of Scenario D to the $T>2$ case. For the nonbankable quantity policy, the optimal policy is $\tilde{q}_{t}=\hat{q}$ in all periods, and expected welfare is $E W_{N B Q}^{M P}=T\left(b_{0}-c_{0}\right)$, where the $M P$ superscript denotes "multi-period." For the non-bankable price policy, the optimal policy is $\tilde{p}_{t}=c_{1}$ in all periods, resulting in a quantity of $q_{t}=\hat{q}-\frac{\theta_{t}}{c_{2}}$, and expected welfare is $E W_{N B P}^{M P}=T\left(b_{0}-c_{0}+\frac{\sigma_{\theta}^{2}}{2 c_{2}^{2}}\left(c_{2}-b_{2}\right)\right)$. The ranking between these two policies is identical to that in the two-period case.

The bankable quantity and bankable price policies are more complicated in the $T>2$ case. Consider first the bankable quantity case. The firm's problem is to choose its production profile $q_{1}, \ldots q_{T}$ to minimize costs in response to the policy variables $\tilde{q}_{1}, \ldots, \tilde{q}_{T}$ and its observed shock values $\theta_{1}, \ldots, \theta_{T}$. The banking and borrowing constraint is $\sum_{t=1}^{T} q_{t}=\sum_{t=1}^{T} \tilde{q}_{t}$. It can be shown that the firm's solution is to produce in each period $q_{t}=\frac{\sum \tilde{q}_{t}}{T}+\frac{\sum \theta_{t}}{T c_{2}}-\frac{\theta_{t}}{c_{2}}$. The first term is just the average allotment; if there were no $\operatorname{cost}$ shocks $\theta$ the firm would perfectly smooth 
production. The second term is the adjustment based on the average cost shock, and the third term is the adjustment based on the period $t$ cost shock.

Given the firm's solution, the planner's solution is to choose the profile of policy variables $\tilde{q}_{1}, \ldots, \tilde{q}_{T}$ to maximize expected welfare, though only the sum $\sum_{t=1}^{T} \tilde{q}_{t}$ matters. Since the planner can true up the sum after observing the second-to-last-period shock, the expectations are taken conditional on all shocks except for $\eta_{T}$ and $\theta_{T}$. The expression for expected welfare that must be maximized includes expectations of random variables across multiple time periods (e.g. $E\left[\eta_{s} \eta_{t}\right]$ for arbitrary $s$ and $t$ ) and is thus generally intractable.

Consider next the bankable price case. The firm's problem is to choose its production profile $q_{1}, \ldots q_{T}$ and the amount of output subject to the price in each period $q_{1}^{B}, \ldots, q_{T}^{B}$ to maximize net revenue in response to the policy variables $\tilde{p}_{1}, \ldots, \tilde{p}_{T}$ and its observed shock values $\theta_{1}, \ldots, \theta_{T}$. That is, $q_{t}$ is the amount actually produced in period $t$, while $q_{t}^{B}$ is the amount for which the firm earns revenue $\tilde{p}_{t} q_{t}^{B}$ in period $t$ after banking or borrowing. The banking and borrowing constraint is $\sum_{t=1}^{T} q_{t}=\sum_{t=1}^{T} q_{t}^{B}$. Obviously the firm will choose to earn all revenue in the period with the highest price; let $\tilde{p}=\max \left\{\tilde{p}_{1}, \ldots, \tilde{p}_{T}\right\}$, then $q_{t}^{B}=\sum_{t=1}^{T} q_{t}$ and $q_{s}^{B}=0 \forall s \neq t$. This yields the firm's solution $q_{t}=\hat{q}+\frac{\tilde{p}-c_{1}-\theta_{t}}{c_{2}}$.

Given the firm's solution, the planner will choose a $\tilde{p}$ in the final period, after observing all of the shock values except for the final period's $\eta_{T}$ and $\theta_{T}$, to maximize expected welfare. As with the solution for the optimal bankable quantity policy, the solution here is generally intractable.

Though closed-form solutions are not presented here for bankable policies in the $T>2$ case, the intuition from the two-period model remains. With policy updating possible, the 
planner will wait until the last period to use all available information before setting the binding policy variable. ${ }^{10}$

\section{Simulations}

I provide numerical simulations of the analytical results presented from scenario D to assist in the interpretation of the effects that have been identified. Several parameter values are taken from the previous literature when applying the model to the case of regulating carbon dioxide to combat climate change. From Pizer and Prest (2016), I set the marginal benefit slope $b_{2}=0 \$ /$ ton $^{2}$ and the marginal cost slope $c_{2}=1.6 \times 10^{-7} \$ /$ ton $^{2}$. Pizer and Prest (2016) use $56(\$ / \text { ton })^{2}$ as the variance of the benefit shock $\sigma_{\eta}^{2}$. For the variance of the cost shock $\sigma_{\theta}^{2}$, I use $169(\$ / \text { ton })^{2}$, which was used by Newell and Pizer (2003). Finally, Newell and Pizer (2003) set the persistence of the cost shock $\rho_{\theta}=0.8$. All of these parameter values are the base-case values. I could not find a source for the persistence of the benefit shock $\rho_{\eta}$, which is absent in Newell and Pizer's (2003) model.

Regardless of the value of $\rho_{\eta}$, at these base-case values the results from scenario D demonstrate that a bankable price policy always dominates the other three policies. Because $c_{2}>b_{2}$, prices dominate quantities, and equation 26 demonstrates that bankable prices always dominate non-bankable prices. I use equation 26 (or equation 25) to evaluate the dollar value of the expected gain from bankable prices relative to non-bankable prices. This value depends on $\rho_{\eta}$ and is higher with a higher value of $\rho_{\eta}$. It ranges from $\$ 88$ million when $\rho_{\eta}=0$ to $\$ 175$ million when $\rho_{\eta}=1$. Since this is a two-period model, and that is the total expected welfare

\footnotetext{
${ }^{10}$ A model with infinite periods may produce more complications - in particular a lack of an equilibrium due to the fact that the firm may keep putting off paying any taxes indefinitely.
} 
benefit over both period, the annual welfare gain of a bankable price policy relative to a nonbankable price policy ranges from $\$ 44$ million to $\$ 88$ million. By comparison, the annual welfare gain of a non-bankable price policy relative to a non-bankable quantity policy is $\$ 528$ million (independent of $\rho_{\eta}$ ). Thus, the gain in adding bankability to a price policy is about an order of magnitude smaller than the gain in moving from a quantity to a price policy.

While this holds for the base case, for other parameter values the gain in moving from non-bankable prices to bankable prices can be even larger than the gain in moving from nonbankable quantities to non-bankable prices. For instance, when the variances of the cost and benefit shock are switched for each other compared to the base case (so that $\sigma_{\eta}^{2}=169(\$ / \text { ton })^{2}$, greater than $\left.\sigma_{\theta}^{2}=56(\$ / \text { ton })^{2}\right)$, then the annual welfare gain of a bankable price policy relative to a non-bankable price policy ranges from $\$ 132$ million to $\$ 264$ million; the annual welfare gain of a non-bankable price policy relative to a non-bankable quantity policy is $\$ 175$ million.

The base-case parameters provide an unambiguous dominance of bankable prices, so I consider an alternate parameterization in which this is not so. I assume that all the parameters are identical to the base case except that I allow the marginal benefit slope $b_{2}$ to vary from 0 (the base case) to $3.2 \times 10^{-7} \$ /$ ton $^{2}$, which is twice the marginal cost slope $c_{2}$ (ensuring a region where prices dominate and a region where quantities dominate). I also allow the persistence of the benefit shock $\rho_{\eta}$, for which there is no base-case value, to vary from 0 to 1.

Figure 1 presents simulation results from this alternative parameterization. The two parameters $\rho_{\eta}$ and $b_{2}$ are varied along the two dimensions. The figure displays which of the policy options dominates: red when bankable prices dominates, blue when bankable quantities dominates, and green when non-bankable quantities dominates. 
Whenever $b_{2}<c_{2}=1.6 \times 10^{-7} \$ /$ ton $^{2}$, bankable prices strictly dominates. When $b_{2}>c_{2}$, quantities dominate prices, but the ranking between bankable and non-bankable quantities can vary. In fact, the figure demonstrates that non-bankable quantities dominate only for the highest values of $b_{2}$ (at least $2.88 \times 10^{-7} \$ / \operatorname{ton}^{2}$, almost double $c_{2}$ ). This corresponds to the fact that the second term in equation 23 is strictly positive, so must be kept small in magnitude for non-bankable quantities to dominate.

\section{Conclusion}

I extend the literature comparing prices, quantities, and bankable quantities by developing a model that allows also for bankable prices. The advantage that bankability offers to a quantity policy can also be extended to a price policy. Whether or not bankability offers an advantage depends on both the relative slopes of the marginal cost and benefit curves and on the correlation across periods between the shock values. All else equal, bankability tends to offer an advantage, but that advantage may be dominated by another effect favoring non-bankability.

While the model is very simple, it confirms the intuition that the advantage afforded by banking is not unique to quantity instruments. Several assumptions can be dropped to see how bankable and non-bankable prices and quantities compare under alternative scenarios. For example, the social benefit of the output can be a stock rather than a flow, as would be the case for a stock pollutant like carbon dioxide. ${ }^{11}$ Uncertainty could be added on the firm's part - for example, the firm might only observe its second-period shock values after the first period. The policymaker may be allowed to modify the trading ratio between periods, or be allowed to set nonlinear policies. The model could be extended to consider policies that combine a price and

\footnotetext{
${ }^{11}$ See e.g. Hoel and Karp (2002) or Pizer (2002).
} 
quantity instrument (Pizer 2002, Burtraw et al. 2018) or political economy frictions (Weitzman 2017).

The policy implications of this paper are in a sense obvious - I identify cases in which allowing bankable prices can be welfare-improving. However, the real-world application may be questionable: is a bankable price policy feasible? Bankable quantity policies have been proven feasible, since most existing cap-and-trade markets have some provisions for intertemporal trading. ${ }^{12}$ I am not aware of any emissions tax policies that offer any form of intertemporal trading, but intertemporal trading is allowed for other types of taxes. For example, with a traditional Individual Retirement Account (IRA), earnings and gains are taxed not when earned but when distributed - the taxpayer gets to defer payment to a later period, with limits. A tax loss carryforward is a tax policy that allows capital losses to be carried forward to future tax years to offset capital gains in those years. Shon and Veliotis (2010) find evidence that individuals strategically pre-pay deductible state income taxes to reduce their federal tax liability - what is called "the December effect." Corporations have the option of accelerated depreciation for their fixed assets, to reduce their tax liability. Given these real-world examples of bankable taxes, it seems feasible that a provision for bankability could be worked in to an emissions pricing policy. This paper demonstrates when it would be efficient to do so.

\footnotetext{
${ }^{12}$ See Table 1 in Hasegawa and Salant (2014) - all six policies presented offer some form of banking or borrowing.
} 


\section{References}

Burtraw, Dallas, Charlie Holt, Karen Palmer, Anthony Paul, and William Shobe. Quantities with Prices. RFF Working Paper 18-08, 2018.

Chevallier, Julien. "Banking and borrowing in the EU ETS: A review of economic modelling, current provisions and prospects for future design." Journal of Economic Surveys 26, no. 1 (2012): 157-176.

Fell, Harrison, Ian A. MacKenzie, and William A. Pizer. "Prices versus quantities versus bankable quantities." Resource and Energy Economics 34, no. 4 (2012): 607-623.

Hasegawa, Makoto, and Stephen Salant. "Cap-and-trade programs under delayed compliance: Consequences of interim injections of permits." Journal of Public Economics 119 (2014): 24-34.

Hoel, Michael, and Larry Karp. "Taxes versus quotas for a stock pollutant." Resource and Energy Economics 24, no. 4 (2002): 367-384.

Kollenberg, Sascha, and Luca Taschini. "Emissions trading systems with cap adjustments." Journal of Environmental Economics and Management 80 (2016): 20-36.

Krysiak, Frank C., and Iris Maria Oberauner. "Environmental policy à la carte: Letting firms choose their regulation." Journal of Environmental Economics and Management 60, no. 3 (2010): 221-232.

Newell, Richard G., and William A. Pizer. "Regulating stock externalities under uncertainty." Journal of environmental economics and management 45, no. 2 (2003): 416-432.

Pizer, William A. "Combining price and quantity controls to mitigate global climate change." Journal of public economics 85, no. 3 (2002): 409-434.

Pizer, William A., and Brian Prest. Prices versus Quantities with Policy Updating. No. w22379. National Bureau of Economic Research, 2016.

Shon, John, and Stanley Veliotis. "Is There a December Effect? Strategic Prepayments of Deductible State Income Tax." Journal of the American Taxation Association 32, no. 2 (2010): 53-71.

Weitzman, Martin L. "Prices vs. quantities." The review of economic studies 41, no. 4 (1974): 477-491.

Weitzman, Martin L. "Voting on prices vs. voting on quantities in a World Climate Assembly." Research in Economics 71, no. 2 (2017): 199-211. 
Weitzman, Martin L. Prices or quantities dominate banking and borrowing. No. 24218. National Bureau of Economic Research, 2018.

Williams, Roberton. Prices vs. quantities vs. tradable quantities. No. w9283. National Bureau of Economic Research, 2002. 


\section{Figure 1 - Policy Simulations}

\section{REGIONS OF POLICY DOMINANCE}

3.2E-07 Non-Bankable

Quantity

$2.4 \mathrm{E}-07$

$b_{2} \quad 1.6 \mathrm{E}-07$

Bankable Quantity

\section{Bankable Price}

$8 \mathrm{E}-08$

0.1

0.2

0.3

0.4

0.5

0.6

0.7

0.8

0.9

$\rho_{\eta}$

Note: This picture displays which of the four policy options dominates for each value of $\rho_{\eta} \in[0,1]$ and $b_{2} \in\left[0,3.2 \times 10^{-7}\right]$. All other parameter values are kept at the base case values described in the text. 
Table 1 - Expected Welfare (Relative to Non-Bankable Quantities)

\begin{tabular}{|c|c|c|c|c|}
\hline & $\begin{array}{c}\text { Non-Bankable } \\
\text { Quantities }\end{array}$ & $\begin{array}{l}\text { Non-Bankable } \\
\text { Prices }\end{array}$ & Bankable Quantities & Bankable Prices \\
\hline $\begin{array}{c}\text { Scenario } \\
\text { A }\end{array}$ & 0 & $\frac{\sigma_{\theta}^{2}}{c_{2}^{2}}\left(c_{2}-b_{2}\right)$ & 0 & $\frac{\sigma_{\theta}^{2}}{c_{2}^{2}}\left(c_{2}-b_{2}\right)$ \\
\hline $\begin{array}{c}\text { Scenario } \\
\text { B }\end{array}$ & $\frac{1}{2}\left(\frac{\sigma_{\eta}^{2}+\sigma_{\theta}^{2}}{b_{2}+c_{2}}\right)$ & $\begin{array}{l}\frac{1}{2}\left(\frac{\sigma_{\eta}^{2}+\sigma_{\theta}^{2}}{b_{2}+c_{2}}\right) \\
+\frac{\sigma_{\theta}^{2}}{2 c_{2}^{2}}\left(c_{2}-b_{2}\right)\end{array}$ & $\left(\frac{\sigma_{\eta}^{2}+\sigma_{\theta}^{2}}{b_{2}+c_{2}}\right)$ & $\left(\frac{\sigma_{\eta}^{2}+\sigma_{\theta}^{2}}{b_{2}+c_{2}}\right)$ \\
\hline $\begin{array}{c}\text { Scenario } \\
\text { C }\end{array}$ & 0 & $\begin{array}{l}\frac{\sigma_{\theta}^{2}}{2 c_{2}^{2}}\left(\rho_{\theta}^{2}+2\right)\left(c_{2}\right. \\
\left.-b_{2}\right)\end{array}$ & $\frac{\sigma_{\theta}^{2}}{4 c_{2}^{2}}\left(\rho_{\theta}^{2}-2 \rho_{\theta}+2\right)\left(c_{2}-b_{2}\right)$ & $\frac{\sigma_{\theta}^{2}}{2 c_{2}^{2}}\left(\rho_{\theta}^{2}+2\right)\left(c_{2}-b_{2}\right)$ \\
\hline \multirow[t]{2}{*}{$\begin{array}{c}\text { Scenario } \\
\text { D }\end{array}$} & & \multirow[b]{2}{*}{$\begin{array}{l}\frac{1}{2} \frac{\rho_{\eta}^{2} \sigma_{\eta}^{2}+\rho_{\theta}^{2} \sigma_{\theta}^{2}}{b_{2}+c_{2}} \\
+\frac{\sigma_{\theta}^{2}}{c_{2}^{2}}\left(c_{2}-b_{2}\right)\end{array}$} & $\underline{1}\left(1+\rho_{\eta}\right)^{2} \sigma_{\eta}^{2}+\left(1+\rho_{\theta}\right)^{2} \sigma_{\theta}^{2}$ & $1\left(1+\rho_{\eta}\right)^{2} \sigma_{\eta}^{2}+\left(1+\rho_{\theta}\right)^{2} \sigma_{\theta}^{2}$ \\
\hline & $\frac{1}{2} \frac{\rho_{\eta}^{2} \sigma_{\eta}^{2}+\rho_{\theta}^{2} \sigma_{\theta}^{2}}{b_{2}+c_{2}}$ & & $\begin{array}{l}\overline{4} \\
\quad b_{2}+c_{2} \\
+\frac{\sigma_{\theta}^{2}}{4 c_{2}^{2}}\left(c_{2}-b_{2}\right)(2 \\
\left.+\rho_{\theta}^{2}-2 \rho_{\theta}\right)\end{array}$ & $\begin{array}{l}\overline{4} \\
\quad b_{2}+c_{2} \\
+\frac{\sigma_{\theta}^{2}}{4 c_{2}^{2}}\left(c_{2}-b_{2}\right)(3 \\
\left.+\rho_{\theta}^{2}-2 \rho_{\theta}\right)\end{array}$ \\
\hline
\end{tabular}

Note: This table presents the expected welfare under each policy, relative to the expected welfare under non-bankable prices in Scenario A. That is, each element in the table is the corresponding expression for $E W$ minus the term $2\left(b_{0}-c_{0}\right)$. 


\section{Appendix}

\section{A.1 Proofs of results in text}

\section{A.1.a Scenario A}

First Best

The first-best outcome is the solution to

$$
\max _{q_{1}, q_{2}} B\left(\tilde{q}_{1}, \eta\right)-C\left(\tilde{q}_{1}, \theta\right)+B\left(\tilde{q}_{2}, \eta\right)-C\left(\tilde{q}_{2}, \theta\right)
$$

that is,

$$
\begin{aligned}
\max _{q_{1}, q_{2}} b_{0}+\left(b_{1}\right. & +\eta)\left(q_{1}-\hat{q}\right)-\frac{b_{2}}{2}\left(q_{1}-\hat{q}\right)^{2}-c_{0}-\left(c_{1}+\theta\right)\left(q_{1}-\hat{q}\right)-\frac{c_{2}}{2}\left(q_{1}-\hat{q}\right)^{2}+b_{0} \\
& +\left(b_{1}+\eta\right)\left(q_{2}-\hat{q}\right)-\frac{b_{2}}{2}\left(q_{2}-\hat{q}\right)^{2}-c_{0}-\left(c_{1}+\theta\right)\left(q_{2}-\hat{q}\right)-\frac{c_{2}}{2}\left(q_{2}-\hat{q}\right)^{2}
\end{aligned}
$$

The derivative with respect to $q_{1}$ is

$$
\left(b_{1}+\eta\right)-b_{2}\left(q_{1}-\hat{q}\right)-\left(c_{1}+\theta\right)-c_{2}\left(q_{1}-\hat{q}\right) .
$$

Setting this equal to zero (noting that $b_{1}=c_{1}$ ) and solving for $q_{1}$ yields the solution $q_{1}=\hat{q}+$

$\frac{\eta-\theta}{b_{2}+c_{2}}$. The derivative with respect to $q_{2}$ is identical except that $q_{2}$ appears in it, therefore $q_{2}=$ $\hat{q}+\frac{\eta-\theta}{b_{2}+c_{2}}$ also.

Non-bankable quantity

Under the non-bankable quantity policy, the planner's problem is to maximize expected utility, not knowing the values of the shocks:

$$
\begin{gathered}
\max _{q_{1}, q_{2}} E\left[b_{0}+\left(b_{1}+\eta\right)\left(q_{1}-\hat{q}\right)-\frac{b_{2}}{2}\left(q_{1}-\hat{q}\right)^{2}-c_{0}-\left(c_{1}+\theta\right)\left(q_{1}-\hat{q}\right)-\frac{c_{2}}{2}\left(q_{1}-\hat{q}\right)^{2}+b_{0}\right. \\
\left.+\left(b_{1}+\eta\right)\left(q_{2}-\hat{q}\right)-\frac{b_{2}}{2}\left(q_{2}-\hat{q}\right)^{2}-c_{0}-\left(c_{1}+\theta\right)\left(q_{2}-\hat{q}\right)-\frac{c_{2}}{2}\left(q_{2}-\hat{q}\right)^{2}\right]
\end{gathered}
$$

This becomes 


$$
\begin{gathered}
\max _{q_{1}, q_{2}} b_{0}+b_{1}\left(q_{1}-\hat{q}\right)-\frac{b_{2}}{2}\left(q_{1}-\hat{q}\right)^{2}-c_{0}-c_{1}\left(q_{1}-\hat{q}\right)-\frac{c_{2}}{2}\left(q_{1}-\hat{q}\right)^{2}+b_{0}+b_{1}\left(q_{2}-\hat{q}\right) \\
-\frac{b_{2}}{2}\left(q_{2}-\hat{q}\right)^{2}-c_{0}-c_{1}\left(q_{2}-\hat{q}\right)-\frac{c_{2}}{2}\left(q_{2}-\hat{q}\right)^{2}
\end{gathered}
$$

Taking the first-order condition with respect to $q_{1}$ yields $q_{1}=\hat{q}$, and likewise for $q_{2}: q_{2}=\hat{q}$.

Given this policy, the expected welfare is expected net benefits evaluated at $q_{1}=q_{2}=\hat{q}$.

Expected welfare is

$$
\begin{gathered}
E\left[b_{0}+\left(b_{1}+\eta\right)\left(q_{1}-\hat{q}\right)-\frac{b_{2}}{2}\left(q_{1}-\hat{q}\right)^{2}-c_{0}-\left(c_{1}+\theta\right)\left(q_{1}-\hat{q}\right)-\frac{c_{2}}{2}\left(q_{1}-\hat{q}\right)^{2}+b_{0}\right. \\
\left.\quad+\left(b_{1}+\eta\right)\left(q_{2}-\hat{q}\right)-\frac{b_{2}}{2}\left(q_{2}-\hat{q}\right)^{2}-c_{0}-\left(c_{1}+\theta\right)\left(q_{2}-\hat{q}\right)-\frac{c_{2}}{2}\left(q_{2}-\hat{q}\right)^{2}\right]
\end{gathered}
$$

In this expression, all terms with $q_{1}-\hat{q}$ or $q_{2}-\hat{q}$ become zero, so $E W_{N B Q}^{A}=2\left(b_{0}-c_{0}\right)$.

Non-bankable price

Next consider the non-bankable price policy. Given a set of policies $\left\{\tilde{p}_{1}, \tilde{p}_{2}\right\}$, the firm's problem is

$$
\begin{aligned}
\max _{q_{1}, q_{2}} \tilde{p}_{1} q_{1}-c_{0} & -\left(c_{1}+\theta\right)\left(q_{1}-\hat{q}\right)-\frac{c_{2}}{2}\left(q_{1}-\hat{q}\right)^{2}+\tilde{p}_{2} q_{2}-c_{0}-\left(c_{1}+\theta\right)\left(q_{2}-\hat{q}\right) \\
& -\frac{c_{2}}{2}\left(q_{2}-\hat{q}\right)^{2}
\end{aligned}
$$

The first-order condition with respect to $q_{1}$ is

$$
\tilde{p}_{1}-\left(c_{1}+\theta\right)-c_{2}\left(q_{1}-\hat{q}\right)=0
$$

Solving this for $q_{1}$ yields $q_{1}=\hat{q}+\frac{\tilde{p}_{1}-c_{1}-\theta}{c_{2}}$. The first-order condition with respect to $q_{2}$ is identical except for replacing $q_{1}$ with $q_{2}$, so $q_{2}=\hat{q}+\frac{\tilde{p}_{2}-c_{1}-\theta}{c_{2}}$. Given this response, the planner sets the prices to maximize expected net benefits: 


$$
\begin{array}{r}
\max _{\tilde{p}_{1}, \tilde{p}_{2}} E\left[b_{0}+\left(b_{1}+\eta\right)\left(q_{1}-\hat{q}\right)-\frac{b_{2}}{2}\left(q_{1}-\hat{q}\right)^{2}-c_{0}-\left(c_{1}+\theta\right)\left(q_{1}-\hat{q}\right)-\frac{c_{2}}{2}\left(q_{1}-\hat{q}\right)^{2}+b_{0}\right. \\
\left.+\left(b_{1}+\eta\right)\left(q_{2}-\hat{q}\right)-\frac{b_{2}}{2}\left(q_{2}-\hat{q}\right)^{2}-c_{0}-\left(c_{1}+\theta\right)\left(q_{2}-\hat{q}\right)-\frac{c_{2}}{2}\left(q_{2}-\hat{q}\right)^{2}\right]
\end{array}
$$

Such that $q_{1}=\hat{q}+\frac{\tilde{p}_{1}-c_{1}-\theta}{c_{2}}$ and $q_{2}=\hat{q}+\frac{\tilde{p}_{2}-c_{1}-\theta}{c_{2}}$. The maximization problem becomes

$$
\begin{aligned}
\max _{\tilde{p}_{1}, \tilde{p}_{2}} E\left[b_{0}+\right. & \left(b_{1}+\eta\right)\left(\frac{\tilde{p}_{1}-c_{1}-\theta}{c_{2}}\right)-\frac{b_{2}}{2}\left(\frac{\tilde{p}_{1}-c_{1}-\theta}{c_{2}}\right)^{2}-c_{0}-\left(c_{1}+\theta\right)\left(\frac{\tilde{p}_{1}-c_{1}-\theta}{c_{2}}\right) \\
& -\frac{c_{2}}{2}\left(\frac{\tilde{p}_{1}-c_{1}-\theta}{c_{2}}\right)^{2}+b_{0}+\left(b_{1}+\eta\right)\left(\frac{\tilde{p}_{2}-c_{1}-\theta}{c_{2}}\right)-\frac{b_{2}}{2}\left(\frac{\tilde{p}_{2}-c_{1}-\theta}{c_{2}}\right)^{2}-c_{0} \\
& \left.-\left(c_{1}+\theta\right)\left(\frac{\tilde{p}_{2}-c_{1}-\theta}{c_{2}}\right)-\frac{c_{2}}{2}\left(\frac{\tilde{p}_{2}-c_{1}-\theta}{c_{2}}\right)^{2}\right]
\end{aligned}
$$

Given that $E[\theta]=E[\eta]=E[\theta \eta]=0$, and $E\left[\theta^{2}\right]=\sigma_{\theta}^{2}$, the problem can be written as

$$
\begin{aligned}
\max _{\tilde{p}_{1}, \tilde{p}_{2}}\left[b_{0}+b_{1}\right. & \left(\frac{\tilde{p}_{1}-c_{1}}{c_{2}}\right)-\frac{b_{2}}{2}\left(\frac{\tilde{p}_{1}-c_{1}}{c_{2}}\right)^{2}-\frac{b_{2}}{2} \frac{\sigma_{\theta}^{2}}{c_{2}^{2}}-c_{0}-c_{1}\left(\frac{\tilde{p}_{1}-c_{1}}{c_{2}}\right)+\frac{\sigma_{\theta}^{2}}{c_{2}}-\frac{c_{2}}{2}\left(\frac{\tilde{p}_{1}-c_{1}}{c_{2}}\right)^{2} \\
& -\frac{c_{2}}{2} \frac{\sigma_{\theta}^{2}}{c_{2}^{2}}+b_{0}+b_{1}\left(\frac{\tilde{p}_{2}-c_{1}}{c_{2}}\right)-\frac{b_{2}}{2}\left(\frac{\tilde{p}_{2}-c_{1}}{c_{2}}\right)^{2}-\frac{b_{2}}{2} \frac{\sigma_{\theta}^{2}}{c_{2}^{2}}-c_{0}-c_{1}\left(\frac{\tilde{p}_{2}-c_{1}}{c_{2}}\right)+\frac{\sigma_{\theta}^{2}}{c_{2}} \\
& \left.-\frac{c_{2}}{2}\left(\frac{\tilde{p}_{2}-c_{1}}{c_{2}}\right)^{2}-\frac{c_{2}}{2} \frac{\sigma_{\theta}^{2}}{c_{2}^{2}}\right]
\end{aligned}
$$

The first-order condition for choice of $\tilde{p}_{1}$ is

$$
\frac{b_{1}}{c_{2}}-\frac{b_{2}}{2 c_{2}^{2}} 2\left(\tilde{p}_{1}-c_{1}\right)-\frac{c_{1}}{c_{2}}+\frac{1}{2 c_{2}} 2\left(\tilde{p}_{1}-c_{1}\right)=0
$$

Solving this yields $\tilde{p}_{1}=c_{1}$. The first-order condition for $\tilde{p}_{2}$ is symmetric, so $\tilde{p}_{2}=c_{1}$. Given these policies, the firm will choose to produce $q_{1}=q_{2}=\hat{q}-\frac{\theta}{c_{2}}$ in each period, from the solution to its maximization problem found above. Substitute these values for $q_{1}$ and $q_{2}$ into the expression for expected welfare 


$$
\begin{gathered}
E\left[b_{0}+\left(b_{1}+\eta\right)\left(q_{1}-\hat{q}\right)-\frac{b_{2}}{2}\left(q_{1}-\hat{q}\right)^{2}-c_{0}-\left(c_{1}+\theta\right)\left(q_{1}-\hat{q}\right)-\frac{c_{2}}{2}\left(q_{1}-\hat{q}\right)^{2}+b_{0}\right. \\
\left.+\left(b_{1}+\eta\right)\left(q_{2}-\hat{q}\right)-\frac{b_{2}}{2}\left(q_{2}-\hat{q}\right)^{2}-c_{0}-\left(c_{1}+\theta\right)\left(q_{2}-\hat{q}\right)-\frac{c_{2}}{2}\left(q_{2}-\hat{q}\right)^{2}\right] \\
=E\left[b_{0}+\left(b_{1}+\eta\right)\left(-\frac{\theta}{c_{2}}\right)-\frac{b_{2}}{2}\left(-\frac{\theta}{c_{2}}\right)^{2}-c_{0}-\left(c_{1}+\theta\right)\left(-\frac{\theta}{c_{2}}\right)-\frac{c_{2}}{2}\left(-\frac{\theta}{c_{2}}\right)^{2}+b_{0}\right. \\
\left.+\left(b_{1}+\eta\right)\left(-\frac{\theta}{c_{2}}\right)-\frac{b_{2}}{2}\left(-\frac{\theta}{c_{2}}\right)^{2}-c_{0}-\left(c_{1}+\theta\right)\left(-\frac{\theta}{c_{2}}\right)-\frac{c_{2}}{2}\left(-\frac{\theta}{c_{2}}\right)^{2}\right] \\
=b_{0}-\frac{b_{2}}{2 c_{2}^{2}} \sigma_{\theta}^{2}-c_{0}+\frac{\sigma_{\theta}^{2}}{c_{2}}-\frac{c_{2}}{2 c_{2}^{2}} \sigma_{\theta}^{2}+b_{0}-\frac{b_{2}}{2 c_{2}^{2}} \sigma_{\theta}^{2}-c_{0}+\frac{\sigma_{\theta}^{2}}{c_{2}}-\frac{c_{2}}{2 c_{2}^{2}} \sigma_{\theta}^{2} \\
=2\left(b_{0}-c_{0}\right)+\frac{\sigma_{\theta}^{2}}{c_{2}^{2}}\left(c_{2}-b_{2}\right)
\end{gathered}
$$

Bankable quantity

The firm's problem is

$$
\begin{gathered}
\max _{q_{1}, q_{2}, B}-C\left(q_{1}, \theta\right)-C\left(q_{2}, \theta\right) \\
\text { s.t. } q_{1}=\tilde{q}_{1}-B, q_{2}=\tilde{q}_{2}+B \\
\max _{q_{1}, q_{2}, B}-\left[c_{0}+\left(c_{1}+\theta\right)\left(\tilde{q}_{1}-B-\hat{q}\right)+\frac{c_{2}}{2}\left(\tilde{q}_{1}-B-\hat{q}\right)^{2}+c_{0}+\left(c_{1}+\theta\right)\left(\tilde{q}_{2}+B-\hat{q}\right)+\right. \\
\left.\frac{c_{2}}{2}\left(\tilde{q}_{2}+B-\hat{q}\right)^{2}\right]
\end{gathered}
$$

The first-order condition with respect to $B$ yields $B=\frac{\tilde{q}_{1}-\tilde{q}_{2}}{2}$. This implies $q_{1}=\tilde{q}_{1}-B=\frac{\tilde{q}_{1}+\tilde{q}_{2}}{2}$.

Similarly, $q_{2}=\tilde{q}_{2}+B=\frac{\tilde{q}_{1}+\tilde{q}_{2}}{2}$. Let $\tilde{q}_{1}+\tilde{q}_{2} \equiv \tilde{q}$. Given this response, the planner sets the quantities $\left\{\tilde{q}_{1}, \tilde{q}_{2}\right\}$ to maximize expected net benefits:

$$
\begin{array}{r}
\max _{\tilde{q}} E\left[b_{0}+\left(b_{1}+\eta\right)\left(\frac{\tilde{q}}{2}-\hat{q}\right)-\frac{b_{2}}{2}\left(\frac{\tilde{q}}{2}-\hat{q}\right)^{2}-c_{0}-\left(c_{1}+\theta\right)\left(\frac{\tilde{q}}{2}-\hat{q}\right)-\frac{c_{2}}{2}\left(\frac{\tilde{q}}{2}-\hat{q}\right)^{2}+b_{0}\right. \\
\left.+\left(b_{1}+\eta\right)\left(\frac{\tilde{q}}{2}-\hat{q}\right)-\frac{b_{2}}{2}\left(\frac{\tilde{q}}{2}-\hat{q}\right)^{2}-c_{0}-\left(c_{1}+\theta\right)\left(\frac{\tilde{q}}{2}-\hat{q}\right)-\frac{c_{2}}{2}\left(\frac{\tilde{q}}{2}-\hat{q}\right)^{2}\right]
\end{array}
$$


Given that $E[\theta]=E[\eta]=E[\theta \eta]=0$, and $E\left[\theta^{2}\right]=\sigma_{\theta}^{2}$, the problem can be written as

$$
\begin{gathered}
\max _{\tilde{q}}\left[b_{0}+b_{1}\left(\frac{\tilde{q}}{2}-\hat{q}\right)-\frac{b_{2}}{2}\left(\frac{\tilde{q}}{2}-\hat{q}\right)^{2}-c_{0}-c_{1}\left(\frac{\tilde{q}}{2}-\hat{q}\right)-\frac{c_{2}}{2}\left(\frac{\tilde{q}}{2}-\hat{q}\right)^{2}+b_{0}+b_{1}\left(\frac{\tilde{q}}{2}-\hat{q}\right)\right. \\
\left.-\frac{b_{2}}{2}\left(\frac{\tilde{q}}{2}-\hat{q}\right)^{2}-c_{0}-c_{1}\left(\frac{\tilde{q}}{2}-\hat{q}\right)-\frac{c_{2}}{2}\left(\frac{\tilde{q}}{2}-\hat{q}\right)^{2}\right]
\end{gathered}
$$

Simplifying, this becomes

$$
\max _{\tilde{q}} 2\left[b_{0}+b_{1}\left(\frac{\tilde{q}}{2}-\hat{q}\right)-\frac{b_{2}}{2}\left(\frac{\tilde{q}}{2}-\hat{q}\right)^{2}-c_{0}-c_{1}\left(\frac{\tilde{q}}{2}-\hat{q}\right)-\frac{c_{2}}{2}\left(\frac{\tilde{q}}{2}-\hat{q}\right)^{2}\right]
$$

The first-order condition with respect to $\tilde{q}$ yields $\tilde{q}=2 \hat{q}$. Given these policies, the firm will choose to produce $q_{1}=q_{2}=\hat{q}$ in each period, from the solution to its maximization problem found above. Since the quantity produced in each period here is same as under the non-bankable quantity policy, the expected welfare will also be equal: $E W_{B Q}^{A}=E W_{N B Q}^{A}=2\left(b_{0}-c_{0}\right)$.

\section{Bankable price}

The firm faces prices in each period $\tilde{p}_{1}$ and $\tilde{p}_{2}$. It is able to bank some of its output (or emissions) $B$ and pay the price in the following period. Alternatively, if $B<0$, it borrows forward some of its output from the second period and pays for it at the first-period price. The firm's problem thus is

$$
\begin{gathered}
\max _{q_{1}, q_{2}, B} \tilde{p}_{1}\left(q_{1}-B\right)-C\left(q_{1}, \theta\right)+\tilde{p}_{2}\left(q_{2}+B\right)-C\left(q_{2}, \theta\right) \\
\text { s.t. } q_{1}-B \geq 0, q_{2}+B \geq 0
\end{gathered}
$$

The non-negativity constraints come from the fact that the total amount banked cannot be negative in any period. I use the Kuhn-Tucker method to solve this optimization problem with inequality constraints. 


$$
\begin{aligned}
\mathcal{L}\left(q_{1}, q_{2}, B ; \lambda_{1}, \lambda_{2}\right) & =\tilde{p}_{1}\left(q_{1}-B\right)-C\left(q_{1}, \theta\right)+\tilde{p}_{2}\left(q_{2}+B\right)-C\left(q_{2}, \theta\right)+\lambda_{1}\left(q_{1}-B\right)+ \\
\lambda_{2}\left(q_{2}+B\right) & \\
\frac{\partial \mathcal{L}}{\partial q_{1}} & =\tilde{p}_{1}-C^{\prime}\left(q_{1}, \theta\right)+\lambda_{1}=0 \\
\frac{\partial \mathcal{L}}{\partial q_{2}} & =\tilde{p}_{2}-C^{\prime}\left(q_{2}, \theta\right)+\lambda_{2}=0 \\
\frac{\partial \mathcal{L}}{\partial B} & =-\tilde{p}_{1}+\tilde{p}_{2}-\lambda_{1}+\lambda_{2}=0 \\
\frac{\partial \mathcal{L}}{\partial \lambda_{1}} & =q_{1}-B \geq 0, \lambda_{1} \geq 0 \text { and } \lambda_{1}\left(q_{1}-B\right)=0 \\
\frac{\partial \mathcal{L}}{\partial \lambda_{2}} & =q_{2}+B \geq 0, \lambda_{2} \geq 0 \text { and } \lambda_{2}\left(q_{2}+B\right)=0
\end{aligned}
$$

Consider four cases regarding the sign of the non-negativity multipliers $\lambda_{1}$ and $\lambda_{2}$.

\section{Case 1: $\lambda_{1}=\lambda_{2}=0$}

$\operatorname{Using}(3): \tilde{p}_{1}=\tilde{p}_{2}$

Using (1) and (2): $\tilde{p}_{1}=C^{\prime}\left(q_{1}, \theta\right)$ and $\tilde{p}_{2}=C^{\prime}\left(q_{2}, \theta\right)$

Therefore, $C^{\prime}\left(q_{1}, \theta\right)=C^{\prime}\left(q_{2}, \theta\right) \Rightarrow q_{1}=q_{2}$

Using (4): $q_{1} \geq B$; and Using (5): $q_{2} \geq-B$,

$q_{1}=q_{2} \Rightarrow q_{1} \geq B$ and $q_{1} \geq-B \Rightarrow B \in\left[-q_{1}, q_{1}\right]$

Now, $\tilde{p}_{1}=C^{\prime}\left(q_{1}, \theta\right)=\left(c_{1}+\theta\right)+c_{2}\left(\tilde{q}_{1}-B-\hat{q}\right)$

This gives $q_{1}=\hat{q}+\frac{\tilde{p}_{1}-c_{1}-\theta}{c_{2}}=q_{2}$ (by symmetry). Case 1 holds only when the two period's prices are equal to each other.

Case 2: $\lambda_{1}=0, \lambda_{2}>0$

$\operatorname{Using}(1): \tilde{p}_{1}=C^{\prime}\left(\mathrm{q}_{1}, \theta\right) \Rightarrow \mathrm{q}_{1}=\hat{\mathrm{q}}+\frac{\widetilde{\mathrm{p}}_{1}-\mathrm{c}_{1}-\theta}{\mathrm{c}_{2}}$

$\operatorname{Using}(3): \lambda_{2}=\tilde{\mathrm{p}}_{1}-\tilde{\mathrm{p}}_{2}>0 \Rightarrow \tilde{\mathrm{p}}_{1}>\tilde{\mathrm{p}}_{2}$ 
$\operatorname{Using}(2): \tilde{p}_{2}-C^{\prime}\left(q_{2}, \theta\right)+\tilde{p}_{1}-\tilde{p}_{2}=0 \Rightarrow \tilde{p}_{1}=C^{\prime}\left(q_{2}, \theta\right) \Rightarrow q_{2}=q_{1}=\hat{q}+\frac{\widetilde{p}_{1}-c_{1}-\theta}{c_{2}}$

Using (5): $\mathrm{B}=-\mathrm{q}_{2}=-\hat{\mathrm{q}}-\frac{\widetilde{\mathrm{p}}_{1}-\mathrm{c}_{1}-\theta}{\mathrm{c}_{2}}$

Using (4): $q_{1}-B \geq 0 \Rightarrow q_{1}-\left(-q_{2}\right) \geq 0 \Rightarrow q_{1}+q_{2} \geq 0$ which is true.

Case 2 represents the case where $\tilde{p}_{1}>\tilde{p}_{2}$, so that the firm borrows forward all of its secondperiod output $\left(B=-q_{2}\right)$ to get paid the higher period-1 price.

Case 3: $\lambda_{1}>0, \lambda_{2}=0$

$\operatorname{Using}(2): \tilde{p}_{2}=C^{\prime}\left(q_{2}, \theta\right) \Rightarrow \mathrm{q}_{2}=\hat{\mathrm{q}}+\frac{\widetilde{\mathrm{p}}_{2}-\mathrm{c}_{1}-\theta}{\mathrm{c}_{2}}$

$\operatorname{Using}(3): \lambda_{1}=\tilde{\mathrm{p}}_{2}-\tilde{\mathrm{p}}_{1}>0 \Rightarrow \tilde{\mathrm{p}}_{2}>\tilde{\mathrm{p}}_{1}$

$\operatorname{Using}(1): \tilde{p}_{1}-C^{\prime}\left(q_{1}, \theta\right)+\tilde{\mathrm{p}}_{2}-\tilde{\mathrm{p}}_{1}=0 \Rightarrow \tilde{\mathrm{p}}_{2}=\mathrm{C}^{\prime}\left(\mathrm{q}_{1}, \theta\right) \Rightarrow \mathrm{q}_{1}=\mathrm{q}_{2}=\hat{\mathrm{q}}+\frac{\widetilde{\mathrm{p}}_{2}-\mathrm{c}_{1}-\theta}{\mathrm{c}_{2}}$

Using (4): $q_{1}=B$

Case 3 represents the case where $\tilde{p}_{2}>\tilde{p}_{1}$, so that the firm banks all of its first-period output $\left(B=q_{1}\right)$ to get paid the higher period-2 price.

\section{Case 4: $\lambda_{1}>0, \lambda_{2}>0$}

Using (4): $q_{1}=B$

Using (5): $\mathrm{q}_{2}=-\mathrm{B} \Rightarrow \mathrm{q}_{2}=-\mathrm{q}_{1} \Rightarrow \mathrm{q}_{2}+\mathrm{q}_{1}=0$

Using (1): $\tilde{p}_{1}-C^{\prime}\left(q_{1}, \theta\right)+\lambda_{1}=0$

Using $\lambda_{2}$ from (3) and substituting in (2) yields: $\tilde{p}_{1}-C^{\prime}\left(q_{2}, \theta\right)+\lambda_{1}=0$

$\Rightarrow \tilde{p}_{1}-C^{\prime}\left(q_{1}, \theta\right)+\lambda_{1}=\tilde{p}_{1}-C^{\prime}\left(q_{2}, \theta\right)+\lambda_{1}$

$\Rightarrow C^{\prime}\left(q_{1}, \theta\right)=C^{\prime}\left(q_{2}, \theta\right)$

$\Rightarrow C^{\prime}\left(q_{1}, \theta\right)=C^{\prime}\left(-q_{1}, \theta\right)$ since $\mathrm{q}_{2}=-\mathrm{q}_{1}$

$\Rightarrow \mathrm{q}_{1}=-\mathrm{q}_{1}$ 
$\Rightarrow \mathrm{q}_{1}=0$

$\Rightarrow \mathrm{q}_{2}=\mathrm{q}_{1}=0=B$

Using (1): $\lambda_{1}=-\tilde{\mathrm{p}}_{1}+\left(c_{1}+\theta+c_{2}(-\hat{q})\right)>0$

Using (2): $\lambda_{2}=-\tilde{\mathrm{p}}_{2}+\left(c_{2}+\theta+c_{2}(-\hat{q})\right)>0$

Case 4 is thus the trivial case where the both prices are so low that the firm chooses to produce nothing in either period; I ignore this case.

Collecting the results from all four cases yields

$$
q_{1}=q_{2}=\hat{q}+\frac{\max \left\{\tilde{p}_{1}, \tilde{p}_{2}\right\}-c_{1}-\theta}{c_{2}}
$$

The quantity produced in each period here is same as under the non-bankable price policy. So, the expected net benefits will also be equal.

$$
E W_{B P}^{A}=E W_{N B P}^{A}=2\left(b_{0}-c_{0}\right)+\frac{\sigma_{\theta}^{2}}{c_{2}}\left(c_{2}-b_{2}\right)
$$

\section{A.1.b Scenario B}

First Best

Identical to scenario A, ex-post first-best output level is $q_{1}=q_{2}=\hat{q}+\frac{\eta-\theta}{b_{2}+c_{2}}$.

\section{Non-bankable quantity}

The firm has no choice but to produce $\left\{\tilde{q}_{1}, \tilde{q}_{2}\right\}$. The planner's problem is now a twoperiod problem, since it can update its quantity policy after the first period. The second-period problem is:

$$
\max _{\tilde{q}_{2}}\left[b_{0}+\left(b_{1}+\eta\right)\left(\tilde{q}_{2}-\hat{q}\right)-\frac{b_{2}}{2}\left(\tilde{q}_{2}-\hat{q}\right)^{2}-c_{0}-\left(c_{1}+\theta\right)\left(\tilde{q}_{2}-\hat{q}\right)-\frac{c_{2}}{2}\left(\tilde{q}_{2}-\hat{q}\right)^{2}\right]
$$

The first-order condition yields: 


$$
\tilde{q}_{2}=\hat{q}+\frac{\eta-\theta}{b_{2}+c_{2}}
$$

The first period problem is:

$$
\begin{aligned}
& \max _{\tilde{q}_{1}} E\left[b_{0}+\left(b_{1}+\eta\right)\left(\tilde{q}_{1}-\hat{q}\right)-\frac{b_{2}}{2}\left(\tilde{q}_{1}-\hat{q}\right)^{2}-c_{0}-\left(c_{1}+\theta\right)\left(\tilde{q}_{1}-\hat{q}\right)-\frac{c_{2}}{2}\left(\tilde{q}_{1}-\hat{q}\right)^{2}+b_{0}\right. \\
& \left.+\left(b_{1}+\eta\right)\left(\tilde{q}_{2}-\hat{q}\right)-\frac{b_{2}}{2}\left(\tilde{q}_{2}-\hat{q}\right)^{2}-c_{0}-\left(c_{1}+\theta\right)\left(\tilde{q}_{2}-\hat{q}\right)-\frac{c_{2}}{2}\left(\tilde{q}_{2}-\hat{q}\right)^{2}\right] \\
& \max _{\tilde{q}_{1}} E\left[b_{0}+\left(b_{1}+\eta\right)\left(\tilde{q}_{1}-\hat{q}\right)-\frac{b_{2}}{2}\left(\tilde{q}_{1}-\hat{q}\right)^{2}-c_{0}-\left(c_{1}+\theta\right)\left(\tilde{q}_{1}-\hat{q}\right)-\frac{c_{2}}{2}\left(\tilde{q}_{1}-\hat{q}\right)^{2}+b_{0}\right. \\
& -\left(b_{1}+\eta\right)\left(\frac{\eta-\theta}{b_{2}+c_{2}}\right)-\frac{b_{2}}{2}\left(\frac{\eta-\theta}{b_{2}+c_{2}}\right)^{2}-c_{0}+\left(c_{1}+\theta\right)\left(\frac{\eta-\theta}{b_{2}+c_{2}}\right) \\
& \left.-\frac{c_{2}}{2}\left(\frac{\eta-\theta}{b_{2}+c_{2}}\right)^{2}\right] \\
& \max _{\tilde{q}_{1}}\left[b_{0}+b_{1}\left(\tilde{q}_{1}-\hat{q}\right)-\frac{b_{2}}{2}\left(\tilde{q}_{1}-\hat{q}\right)^{2}-c_{0}-c_{1}\left(\tilde{q}_{1}-\hat{q}\right)-\frac{c_{2}}{2}\left(\tilde{q}_{1}-\hat{q}\right)^{2}+b_{0}+\frac{E\left(\eta^{2}\right)}{b_{2}+c_{2}}\right. \\
& -\frac{b_{2}}{2\left(b_{2}+c_{2}\right)^{2}}\left[E\left(\eta^{2}\right)+E\left(\theta^{2}\right)\right]-c_{0}+\frac{E\left(\theta^{2}\right)}{\left(b_{2}+c_{2}\right)} \\
& \left.-\frac{c_{2}}{2\left(b_{2}+c_{2}\right)^{2}}\left[E\left(\eta^{2}\right)+E\left(\theta^{2}\right)\right]\right]
\end{aligned}
$$

The first-order condition with respect to $\tilde{q}_{1}$ is

$$
b_{1}-b_{2}\left(\tilde{q}_{1}-\hat{q}\right)-c_{1}-c_{2}\left(\tilde{q}_{1}-\hat{q}\right)=0
$$

Therefore,

$$
\tilde{q}_{1}=\hat{q}
$$

Substituting these two solutions for $\tilde{q}_{1}$ and $\tilde{q}_{2}$ into the expresstion for expected welfare (note that all of the first-period terms with $\tilde{q}_{1}-\hat{q}$ become zero) yields 


$$
\begin{aligned}
E W_{N B Q}^{B}=b_{0}- & c_{0}+b_{0}+\frac{\sigma_{\eta}^{2}}{b_{2}+c_{2}}-\frac{b_{2}}{2\left(b_{2}+c_{2}\right)^{2}}\left[\sigma_{\eta}^{2}+\sigma_{\theta}^{2}\right]-c_{0}+\frac{\sigma_{\theta}^{2}}{\left(b_{2}+c_{2}\right)} \\
& \quad-\frac{c_{2}}{2\left(b_{2}+c_{2}\right)^{2}}\left[\sigma_{\eta}^{2}+\sigma_{\theta}^{2}\right] \\
= & 2\left(b_{0}-c_{0}\right)+\frac{1}{2\left(b_{2}+c_{2}\right)}\left[\sigma_{\eta}^{2}+\sigma_{\theta}^{2}\right]
\end{aligned}
$$

Non-bankable price

Given a set of policies $\left\{\tilde{p}_{1}, \tilde{p}_{2}\right\}$, the firm's problem is same as in scenario A. Thus, $q_{1}=$ $\hat{q}+\frac{\tilde{p}_{1}-c_{1}-\theta}{c_{2}}$ and $q_{2}=\hat{q}+\frac{\tilde{p}_{2}-c_{1}-\theta}{c_{2}}$. Given this response, the planner's second-period problem is as follows:

$$
\begin{gathered}
\max _{\tilde{p}_{2}}\left[b_{0}+\left(b_{1}+\eta\right)\left(q_{2}-\hat{q}\right)-\frac{b_{2}}{2}\left(q_{2}-\hat{q}\right)^{2}-c_{0}-\left(c_{1}+\theta\right)\left(q_{2}-\hat{q}\right)-\frac{c_{2}}{2}\left(q_{2}-\hat{q}\right)^{2}\right] \\
=\max _{\tilde{p}_{2}}\left[b_{0}+\left(b_{1}+\eta\right)\left(\frac{\tilde{p}_{2}-c_{1}-\theta}{c_{2}}\right)-\frac{b_{2}}{2}\left(\frac{\tilde{p}_{2}-c_{1}-\theta}{c_{2}}\right)^{2}-c_{0}-\left(c_{1}+\theta\right)\left(\frac{\tilde{p}_{2}-c_{1}-\theta}{c_{2}}\right)\right. \\
-\frac{c_{2}}{2}\left(\frac{\tilde{p}_{2}-c_{1}-\theta}{c_{2}}\right)^{2}
\end{gathered}
$$

The first-order condition is

$$
\frac{b_{1}+\eta}{c_{2}}-\frac{b_{2}}{c_{2}}\left(\frac{\tilde{p}_{2}-c_{1}-\theta}{c_{2}}\right)-\frac{c_{1}+\theta}{c_{2}}-\left(\frac{\tilde{p}_{2}-c_{1}-\theta}{c_{2}}\right)=0
$$

Solving this expression gives

$$
\tilde{p}_{2}=c_{1}+\frac{\theta b_{2}+\eta c_{2}}{b_{2}+c_{2}}
$$

This implies that the firm's chosen $q_{2}$ is

$$
q_{2}=\hat{q}+\frac{\eta-\theta}{b_{2}+c_{2}}
$$

The planner's first-period problem is 


$$
\begin{gathered}
\max _{\tilde{p}_{1}} E\left[b_{0}+\left(b_{1}+\eta\right)\left(q_{1}-\hat{q}\right)-\frac{b_{2}}{2}\left(q_{1}-\hat{q}\right)^{2}-c_{0}-\left(c_{1}+\theta\right)\left(q_{1}-\hat{q}\right)-\frac{c_{2}}{2}\left(q_{1}-\hat{q}\right)^{2}+b_{0}\right. \\
\left.+\left(b_{1}+\eta\right)\left(q_{2}-\hat{q}\right)-\frac{b_{2}}{2}\left(q_{2}-\hat{q}\right)^{2}-c_{0}-\left(c_{1}+\theta\right)\left(q_{2}-\hat{q}\right)-\frac{c_{2}}{2}\left(q_{2}-\hat{q}\right)^{2}\right] \\
\text { such that } q_{1}=\hat{q}+\frac{\tilde{p}_{1}-c_{1}-\theta}{c_{2}} \text { and } q_{2}=\hat{q}+\frac{\eta-\theta}{b_{2}+c_{2}} \\
\max _{\tilde{p}_{1}} E\left[b_{0}+\left(b_{1}+\eta\right)\left(\frac{\tilde{p}_{1}-c_{1}-\theta}{c_{2}}\right)-\frac{b_{2}}{2}\left(\frac{\tilde{p}_{1}-c_{1}-\theta}{c_{2}}\right)^{2}-c_{0}-\left(c_{1}+\theta\right)\left(\frac{\tilde{p}_{1}-c_{1}-\theta}{c_{2}}\right)\right. \\
-\frac{c_{2}}{2}\left(\frac{\tilde{p}_{1}-c_{1}-\theta}{c_{2}}\right)^{2}+b_{0}+\left(b_{1}+\eta\right)\left(\frac{\eta-\theta}{b_{2}+c_{2}}\right)-\frac{b_{2}}{2}\left(\frac{\eta-\theta}{b_{2}+c_{2}}\right)^{2}-c_{0} \\
\left.-\left(c_{1}+\theta\right)\left(\frac{\eta-\theta}{b_{2}+c_{2}}\right)-\frac{c_{2}}{2}\left(\frac{\eta-\theta}{b_{2}+c_{2}}\right)^{2}\right]
\end{gathered}
$$

After taking the expectation, the first-order condition with respect to $\tilde{p}_{1}$ yields

$$
\tilde{p}_{1}=c_{1}
$$

The firm's $q_{1}$ is

$$
q_{1}=\hat{q}-\frac{\theta}{c_{2}}
$$

Substituting the firm's choices for $q_{1}$ and $q_{2}$ into expected welfare gives:

$$
\begin{gathered}
E W_{N B P}^{B}=E\left[b_{0}+\left(b_{1}+\eta\right)\left(-\frac{\theta}{c_{2}}\right)-\frac{b_{2}}{2}\left(-\frac{\theta}{c_{2}}\right)^{2}-c_{0}-\left(c_{1}+\theta\right)\left(-\frac{\theta}{c_{2}}\right)-\frac{c_{2}}{2}\left(-\frac{\theta}{c_{2}}\right)^{2}+b_{0}\right. \\
+\left(b_{1}+\eta\right)\left(\frac{\eta-\theta}{b_{2}+c_{2}}\right)-\frac{b_{2}}{2}\left(\frac{\eta-\theta}{b_{2}+c_{2}}\right)^{2}-c_{0}-\left(c_{1}+\theta\right)\left(\frac{\eta-\theta}{b_{2}+c_{2}}\right) \\
\left.-\frac{c_{2}}{2}\left(\frac{\eta-\theta}{b_{2}+c_{2}}\right)^{2}\right] \\
=b_{0}-\frac{b_{2}}{2 c_{2}^{2}} \sigma_{\theta}^{2}-c_{0}+\frac{\sigma_{\theta}^{2}}{c_{2}}-\frac{c_{2}}{2 c_{2}^{2}} \sigma_{\theta}^{2}+b_{0}+\frac{\sigma_{\eta}^{2}}{b_{2}+c_{2}}-\frac{b_{2}}{2\left(b_{2}+c_{2}\right)^{2}}\left[\sigma_{\eta}^{2}+\sigma_{\theta}^{2}\right]-c_{0} \\
+\frac{\sigma_{\theta}^{2}}{\left(b_{2}+c_{2}\right)}-\frac{c_{2}}{2\left(b_{2}+c_{2}\right)^{2}}\left[\sigma_{\eta}^{2}+\sigma_{\theta}^{2}\right]
\end{gathered}
$$


This expression finally simplifies to:

$$
E W_{N B P}^{B}=2\left(b_{0}-c_{0}\right)+\frac{1}{2\left(b_{2}+c_{2}\right)}\left[\sigma_{\eta}^{2}+\sigma_{\theta}^{2}\right]+\frac{\sigma_{\theta}^{2}}{2 c_{2}^{2}}\left(c_{2}-b_{2}\right)
$$

\section{Bankable quantity}

The firm's problem here is the same as the bankable quantity policy under scenario A.

Therefore, $q_{1}=\tilde{q}_{1}-B=\frac{\tilde{q}_{1}+\tilde{q}_{2}}{2}$. Similarly, $q_{2}=\tilde{q}_{2}+B=\frac{\tilde{q}_{1}+\tilde{q}_{2}}{2}$. Let $\tilde{q}_{1}+\tilde{q}_{2} \equiv \tilde{q}$. Given this response, the planner's second period problem becomes

$$
\left.\max _{\tilde{q}} E\left[b_{0}+\left(b_{1}+\eta\right)\left(\frac{\tilde{q}}{2}-\hat{q}\right)-\frac{b_{2}}{2}\left(\frac{\tilde{q}}{2}-\hat{q}\right)^{2}-c_{0}-\left(c_{1}+\theta\right)\left(\frac{\tilde{q}}{2}-\hat{q}\right)-\frac{c_{2}}{2}\left(\frac{\tilde{q}}{2}-\hat{q}\right)^{2}\right]\right]
$$

The first-order condition with respect to $\tilde{q}$ yields

$$
\begin{gathered}
\frac{\tilde{q}}{2}=\hat{q}+\frac{\eta-\theta}{b_{2}+c_{2}} \\
\tilde{q}_{2}=2 \hat{q}+2 \frac{\eta-\theta}{b_{2}+c_{2}}-\tilde{q}_{1}
\end{gathered}
$$

Planner's first period problem would be:

$$
\begin{gathered}
\max _{\tilde{q}}\left[b_{0}+b_{1}\left(\frac{\tilde{q}}{2}-\hat{q}\right)-\frac{b_{2}}{2}\left(\frac{\tilde{q}}{2}-\hat{q}\right)^{2}-c_{0}-c_{1}\left(\frac{\tilde{q}}{2}-\hat{q}\right)-\frac{c_{2}}{2}\left(\frac{\tilde{q}}{2}-\hat{q}\right)^{2}+b_{0}+b_{1}\left(\frac{\tilde{q}}{2}-\hat{q}\right)\right. \\
\left.-\frac{b_{2}}{2}\left(\frac{\tilde{q}}{2}-\hat{q}\right)^{2}-c_{0}-c_{1}\left(\frac{\tilde{q}}{2}-\hat{q}\right)-\frac{c_{2}}{2}\left(\frac{\tilde{q}}{2}-\hat{q}\right)^{2}\right]
\end{gathered}
$$

such that

$$
\tilde{q}_{2}=2 \hat{q}+2 \frac{\eta-\theta}{b_{2}+c_{2}}-\tilde{q}_{1}
$$

and,

$$
\tilde{q}_{1}+\tilde{q}_{2}=\tilde{q}
$$

The firm responds only to the sum $\tilde{q}_{1}+\tilde{q}_{2}$ : 


$$
q_{1}=q_{2}=\frac{\tilde{q}_{1}+\tilde{q}_{2}}{2}=\hat{q}+\frac{\eta-\theta}{b_{2}+c_{2}}
$$

Substituting these values into expected welfare gives

$$
\begin{gathered}
E W_{B Q}^{B}=b_{0}+\left(b_{1}+\eta\right)\left(q_{1}-\hat{q}\right)-\frac{b_{2}}{2}\left(q_{1}-\hat{q}\right)^{2}-c_{0}-\left(c_{1}+\theta\right)\left(q_{1}-\hat{q}\right)-\frac{c_{2}}{2}\left(q_{1}-\hat{q}\right)^{2}+b_{0} \\
+\left(b_{1}+\eta\right)\left(q_{2}-\hat{q}\right)-\frac{b_{2}}{2}\left(q_{2}-\hat{q}\right)^{2}-c_{0}-\left(c_{1}+\theta\right)\left(q_{2}-\hat{q}\right)-\frac{c_{2}}{2}\left(q_{2}-\hat{q}\right)^{2} \\
=2 E\left[b_{0}+\left(b_{1}+\eta\right)\left(\frac{\eta-\theta}{b_{2}+c_{2}}\right)-\frac{b_{2}}{2}\left(\frac{\eta-\theta}{b_{2}+c_{2}}\right)^{2}-c_{0}-\left(c_{1}+\theta\right)\left(\frac{\eta-\theta}{b_{2}+c_{2}}\right)-\frac{c_{2}}{2}\left(\frac{\eta-\theta}{b_{2}+c_{2}}\right)^{2}\right] \\
=2\left[b_{0}+\frac{\sigma_{\eta}^{2}}{b_{2}+c_{2}}-\frac{b_{2}}{2\left(b_{2}+c_{2}\right)^{2}}\left[\sigma_{\eta}^{2}+\sigma_{\theta}^{2}\right]-c_{0}+\frac{\sigma_{\theta}^{2}}{\left(b_{2}+c_{2}\right)}-\frac{c_{2}}{2\left(b_{2}+c_{2}\right)^{2}}\left[\sigma_{\eta}^{2}+\sigma_{\theta}^{2}\right]\right]
\end{gathered}
$$

This expression finally simplifies to:

$$
E W_{B Q}^{B}=2\left(b_{0}-c_{0}\right)+\frac{1}{\left(b_{2}+c_{2}\right)}\left[\sigma_{\eta}^{2}+\sigma_{\theta}^{2}\right]
$$

Therefore,

$$
E W_{B Q}^{B}-E W_{N B Q}^{B}=\frac{1}{2\left(b_{2}+c_{2}\right)}\left[\sigma_{\eta}^{2}+\sigma_{\theta}^{2}\right]
$$

Additionally, $E W_{B Q}^{B}-E W_{N B P}^{B}=2\left(b_{0}-c_{0}\right)+\frac{1}{\left(b_{2}+c_{2}\right)}\left[\sigma_{\eta}^{2}+\sigma_{\theta}^{2}\right]-2\left(b_{0}-c_{0}\right)-$

$$
\frac{1}{2\left(b_{2}+c_{2}\right)}\left[\sigma_{\eta}^{2}+\sigma_{\theta}^{2}\right]-\frac{\sigma_{\theta}^{2}}{2 c_{2}^{2}}\left(c_{2}-b_{2}\right)
$$

On further simplification, we get:

$$
E W_{B Q}^{B}-E W_{N B P}^{B}=\frac{1}{2\left(b_{2}+c_{2}\right)}\left[\sigma_{\eta}^{2}+\sigma_{\theta}^{2}\left(\frac{b_{2}}{c_{2}}\right)^{2}\right]>0
$$

\section{Bankable price}

The firm's response to this policy is the same as its response under scenario A:

$$
q_{1}=q_{2}=\hat{q}+\frac{\max \left\{\tilde{p}_{1}, \tilde{p}_{2}\right\}-c_{1}-\theta}{c_{2}}
$$

Assuming that $\tilde{p}_{2}$ is the binding price, the planner's second-period problem is 


$$
\begin{aligned}
\max _{\tilde{p}_{2}} b_{0}+\left(b_{1}\right. & +\eta)\left(\frac{\tilde{p}_{2}-c_{1}-\theta}{c_{2}}\right)-\frac{b_{2}}{2}\left(\frac{\tilde{p}_{2}-c_{1}-\theta}{c_{2}}\right)^{2}-c_{0}-\left(c_{1}+\theta\right)\left(\frac{\tilde{p}_{2}-c_{1}-\theta}{c_{2}}\right) \\
& -\frac{c_{2}}{2}\left(\frac{\tilde{p}_{2}-c_{1}-\theta}{c_{2}}\right)^{2}
\end{aligned}
$$

The first-order condition with respect to $\tilde{p}_{2}$ yields

$$
\tilde{p}_{2}=c_{1}+\frac{b_{2} \theta+c_{2} \eta}{b_{2}+c_{2}}
$$

Substituting this $\tilde{p}_{2}$ into the firm's choice for $q_{2}$ yields

$$
q_{2}=\hat{q}+\frac{\eta-\theta}{b_{2}+c_{2}}=q_{1}
$$

The first-period price can be anything arbitrary low enough to ensure that it is always lower than the first-period price. Thus, the planner knows that the firm will actually face the second-period price, and it knows that by the second period it will have enough information to set that price to achieve the first-best.

The resulting quantities are identical to those under the bankable price policy, and thus the expected welfare is identical to the expected welfare under that policy.

\section{A.1.C Scenario C}

In this scenario:

$$
\begin{aligned}
& \theta_{2}=\rho_{\theta} \theta_{1}+\varepsilon_{\theta} \\
& \eta_{2}=\rho_{\eta} \eta_{1}+\varepsilon_{\eta}
\end{aligned}
$$

and,

$$
\begin{aligned}
& E\left[\theta_{1}\right]=E\left[\theta_{2}\right]=E\left[\eta_{1}\right]=E\left[\eta_{2}\right]=E\left[\theta_{l} \eta_{m}\right]=0, E\left[\theta_{1}^{2}\right]=\sigma_{\theta}^{2}, E\left[\eta_{1}^{2}\right]=\sigma_{\eta}^{2}, \\
& E\left[\theta_{2}^{2}\right]=\sigma_{\theta}^{2}\left(\rho_{\theta}^{2}+1\right), E\left[\theta_{1} \theta_{2}\right]=\rho_{\theta} \sigma_{\theta}^{2}, E\left[\eta_{2}^{2}\right]=\sigma_{\eta}^{2}\left(\rho_{\eta}^{2}+1\right), E\left[\eta_{1} \eta_{2}\right]=\rho_{\eta} \sigma_{\eta}^{2}
\end{aligned}
$$

First best 
In this scenario, as calculated above in scenarios A and B, the ex-post first-best outcome will be $q_{1}=\hat{q}+\frac{\eta_{1}-\theta_{1}}{b_{2}+c_{2}}$ and $q_{2}=\hat{q}+\frac{\eta_{2}-\theta_{2}}{b_{2}+c_{2}}$. (This is the same as in the previous scenarios, except noting that the shock values differ across periods.)

Non-bankable quantity

The planner's problem here is:

$$
\begin{gathered}
\max _{q_{1}, q_{2}} E\left[b_{0}+\left(b_{1}+\eta_{1}\right)\left(q_{1}-\hat{q}\right)-\frac{b_{2}}{2}\left(q_{1}-\hat{q}\right)^{2}-c_{0}-\left(c_{1}+\theta_{1}\right)\left(q_{1}-\hat{q}\right)-\frac{c_{2}}{2}\left(q_{1}-\hat{q}\right)^{2}+b_{0}\right. \\
\left.+\left(b_{1}+\eta_{2}\right)\left(q_{2}-\hat{q}\right)-\frac{b_{2}}{2}\left(q_{2}-\hat{q}\right)^{2}-c_{0}-\left(c_{1}+\theta_{2}\right)\left(q_{2}-\hat{q}\right)-\frac{c_{2}}{2}\left(q_{2}-\hat{q}\right)^{2}\right]
\end{gathered}
$$

This becomes

$$
\begin{gathered}
\max _{q_{1}, q_{2}} b_{0}+b_{1}\left(q_{1}-\hat{q}\right)-\frac{b_{2}}{2}\left(q_{1}-\hat{q}\right)^{2}-c_{0}-c_{1}\left(q_{1}-\hat{q}\right)-\frac{c_{2}}{2}\left(q_{1}-\hat{q}\right)^{2}+b_{0}+b_{1}\left(q_{2}-\hat{q}\right) \\
-\frac{b_{2}}{2}\left(q_{2}-\hat{q}\right)^{2}-c_{0}-c_{1}\left(q_{2}-\hat{q}\right)-\frac{c_{2}}{2}\left(q_{2}-\hat{q}\right)^{2}
\end{gathered}
$$

Taking the first-order condition with respect to $q_{1}$ yields $q_{1}=\hat{q}$, and likewise for $q_{2}: q_{2}=\hat{q}$.

Given this policy, the expected welfare is expected net benefits evaluated at $q_{1}=q_{2}=\hat{q}$.

Expected welfare is

$$
\begin{aligned}
E\left[b_{0}+\left(b_{1}+\right.\right. & \left.\eta_{1}\right)\left(q_{1}-\hat{q}\right)-\frac{b_{2}}{2}\left(q_{1}-\hat{q}\right)^{2}-c_{0}-\left(c_{1}+\theta_{1}\right)\left(q_{1}-\hat{q}\right)-\frac{c_{2}}{2}\left(q_{1}-\hat{q}\right)^{2}+b_{0} \\
& \left.+\left(b_{1}+\eta_{2}\right)\left(q_{2}-\hat{q}\right)-\frac{b_{2}}{2}\left(q_{2}-\hat{q}\right)^{2}-c_{0}-\left(c_{1}+\theta_{2}\right)\left(q_{2}-\hat{q}\right)-\frac{c_{2}}{2}\left(q_{2}-\hat{q}\right)^{2}\right]
\end{aligned}
$$

In this expression, all terms with $q_{1}-\hat{q}$ or $q_{2}-\hat{q}$ become zero, so $E W_{N B Q}^{C}=2\left(b_{0}-c_{0}\right)$.

Non-bankable price

Given a set of policies $\left\{\tilde{p}_{1}, \tilde{p}_{2}\right\}$, the firm's problem is 


$$
\begin{gathered}
\max _{q_{1}, q_{2}} \tilde{p}_{1} q_{1}-c_{0}-\left(c_{1}+\theta_{1}\right)\left(q_{1}-\hat{q}\right)-\frac{c_{2}}{2}\left(q_{1}-\hat{q}\right)^{2}+\tilde{p}_{2} q_{2}-c_{0}-\left(c_{1}+\theta_{2}\right)\left(q_{2}-\hat{q}\right) \\
-\frac{c_{2}}{2}\left(q_{2}-\hat{q}\right)^{2}
\end{gathered}
$$

The first-order condition with respect to $q_{1}$ is

$$
\tilde{p}_{1}-\left(c_{1}+\theta_{1}\right)-c_{2}\left(q_{1}-\hat{q}\right)=0
$$

Solving this for $q_{1}$ yields $q_{1}=\hat{q}+\frac{\tilde{p}_{1}-c_{1}-\theta_{1}}{c_{2}}$. The first-order condition with respect to $q_{2}$ yields $q_{2}=\hat{q}+\frac{\tilde{p}_{2}-c_{1}-\theta_{2}}{c_{2}}$. Given this response, the planner sets the prices to maximize expected net benefits:

$$
\begin{aligned}
\max _{\tilde{p}_{1}, \tilde{p}_{2}} E\left[b_{0}+\right. & \left(b_{1}+\eta_{1}\right)\left(q_{1}-\hat{q}\right)-\frac{b_{2}}{2}\left(q_{1}-\hat{q}\right)^{2}-c_{0}-\left(c_{1}+\theta_{1}\right)\left(q_{1}-\hat{q}\right)-\frac{c_{2}}{2}\left(q_{1}-\hat{q}\right)^{2}+b_{0} \\
& \left.+\left(b_{1}+\eta_{2}\right)\left(q_{2}-\hat{q}\right)-\frac{b_{2}}{2}\left(q_{2}-\hat{q}\right)^{2}-c_{0}-\left(c_{1}+\theta_{2}\right)\left(q_{2}-\hat{q}\right)-\frac{c_{2}}{2}\left(q_{2}-\hat{q}\right)^{2}\right]
\end{aligned}
$$

such that $q_{1}=\hat{q}+\frac{\tilde{p}_{1}-c_{1}-\theta_{1}}{c_{2}}$ and $q_{2}=\hat{q}+\frac{\tilde{p}_{2}-c_{1}-\theta_{2}}{c_{2}}$

$$
\begin{gathered}
\max _{\tilde{p}_{1}, \tilde{p}_{2}} E\left[b_{0}+\left(b_{1}+\eta_{1}\right)\left(\frac{\tilde{p}_{1}-c_{1}-\theta_{1}}{c_{2}}\right)-\frac{b_{2}}{2}\left(\frac{\tilde{p}_{1}-c_{1}-\theta_{1}}{c_{2}}\right)^{2}-c_{0}-\left(c_{1}+\theta_{1}\right)\left(\frac{\tilde{p}_{1}-c_{1}-\theta_{1}}{c_{2}}\right)\right. \\
-\frac{c_{2}}{2}\left(\frac{\tilde{p}_{1}-c_{1}-\theta_{1}}{c_{2}}\right)^{2}+b_{0}+\left(b_{1}+\eta_{2}\right)\left(\frac{\tilde{p}_{2}-c_{1}-\theta_{2}}{c_{2}}\right)-\frac{b_{2}}{2}\left(\frac{\tilde{p}_{2}-c_{1}-\theta_{2}}{c_{2}}\right)^{2} \\
\left.-c_{0}-\left(c_{1}+\theta_{2}\right)\left(\frac{\tilde{p}_{2}-c_{1}-\theta_{2}}{c_{2}}\right)-\frac{c_{2}}{2}\left(\frac{\tilde{p}_{2}-c_{1}-\theta_{2}}{c_{2}}\right)^{2}\right]
\end{gathered}
$$




$$
\begin{gathered}
\max _{\tilde{p}_{1}, \tilde{p}_{2}}\left[b_{0}+b_{1}\left(\frac{\tilde{p}_{1}-c_{1}}{c_{2}}\right)-\frac{b_{2}}{2}\left(\frac{\tilde{p}_{1}-c_{1}}{c_{2}}\right)^{2}-\frac{b_{2}}{2} \frac{\sigma_{\theta}^{2}}{c_{2}^{2}}-c_{0}-c_{1}\left(\frac{\tilde{p}_{1}-c_{1}}{c_{2}}\right)+\frac{\sigma_{\theta}^{2}}{c_{2}}-\frac{c_{2}}{2}\left(\frac{\tilde{p}_{1}-c_{1}}{c_{2}}\right)^{2}\right. \\
-\frac{c_{2}}{2} \frac{\sigma_{\theta}^{2}}{c_{2}^{2}}+b_{0}+b_{1}\left(\frac{\tilde{p}_{2}-c_{1}}{c_{2}}\right)-\frac{b_{2}}{2}\left(\frac{\tilde{p}_{2}-c_{1}}{c_{2}}\right)^{2}-\frac{b_{2}}{2} \frac{\sigma_{\theta}^{2}\left(\rho_{\theta}^{2}+1\right)}{c_{2}^{2}}-c_{0} \\
\left.-c_{1}\left(\frac{\tilde{p}_{2}-c_{1}}{c_{2}}\right)+\frac{\sigma_{\theta}^{2}\left(\rho_{\theta}^{2}+1\right)}{c_{2}}-\frac{c_{2}}{2}\left(\frac{\tilde{p}_{2}-c_{1}}{c_{2}}\right)^{2}-\frac{c_{2}}{2} \frac{\sigma_{\theta}^{2}\left(\rho_{\theta}^{2}+1\right)}{c_{2}^{2}}\right]
\end{gathered}
$$

The first-order condition for choice of $\tilde{p}_{1}$ is

$$
\frac{b_{1}}{c_{2}}-\frac{b_{2}}{c_{2}^{2}}\left(\tilde{p}_{1}-c_{1}\right)-\frac{c_{1}}{c_{2}}-\frac{1}{c_{2}}\left(\tilde{p}_{1}-c_{1}\right)
$$

Solving this, as in scenario A, yields $\tilde{p}_{1}=c_{1}$. Similarly, the first-order condition with respect to $\tilde{p}_{2}$ yields $\tilde{p}_{2}=c_{1}$. Given these policies, the firm will choose to produce $q_{1}=\hat{q}-\frac{\theta_{1}}{c_{2}}$ and $q_{2}=$ $\hat{q}-\frac{\theta_{2}}{c_{2}}$. Substitute these values for $q_{1}$ and $q_{2}$ into the expression for expected net benefits

$$
\begin{gathered}
E\left[b_{0}+\left(b_{1}+\eta_{1}\right)\left(q_{1}-\hat{q}\right)-\frac{b_{2}}{2}\left(q_{1}-\hat{q}\right)^{2}-c_{0}-\left(c_{1}+\theta_{1}\right)\left(q_{1}-\hat{q}\right)-\frac{c_{2}}{2}\left(q_{1}-\hat{q}\right)^{2}+b_{0}\right. \\
\left.+\left(b_{1}+\eta_{2}\right)\left(q_{2}-\hat{q}\right)-\frac{b_{2}}{2}\left(q_{2}-\hat{q}\right)^{2}-c_{0}-\left(c_{1}+\theta_{2}\right)\left(q_{2}-\hat{q}\right)-\frac{c_{2}}{2}\left(q_{2}-\hat{q}\right)^{2}\right] \\
=E\left[b_{0}+\left(b_{1}+\eta_{1}\right)\left(-\frac{\theta_{1}}{c_{2}}\right)-\frac{b_{2}}{2}\left(-\frac{\theta_{1}}{c_{2}}\right)^{2}-c_{0}-\left(c_{1}+\theta_{1}\right)\left(-\frac{\theta_{1}}{c_{2}}\right)-\frac{c_{2}}{2}\left(-\frac{\theta_{1}}{c_{2}}\right)^{2}+b_{0}\right. \\
\left.+\left(b_{1}+\eta_{2}\right)\left(-\frac{\theta_{2}}{c_{2}}\right)-\frac{b_{2}}{2}\left(-\frac{\theta_{2}}{c_{2}}\right)^{2}-c_{0}-\left(c_{1}+\theta_{2}\right)\left(-\frac{\theta_{2}}{c_{2}}\right)-\frac{c_{2}}{2}\left(-\frac{\theta_{2}}{c_{2}}\right)^{2}\right] \\
=b_{0}-\frac{b_{2}}{2} \frac{\sigma_{\theta}^{2}}{c_{2}^{2}}-c_{0}+\frac{\sigma_{\theta}^{2}}{c_{2}}-\frac{c_{2}}{2} \frac{\sigma_{\theta}^{2}}{c_{2}^{2}}-\frac{c_{2}}{2} \frac{\sigma_{\theta}^{2}}{c_{2}^{2}}+b_{0}-\frac{b_{2}}{2} \frac{\sigma_{\theta}^{2}\left(\rho_{\theta}^{2}+1\right)}{c_{2}^{2}}-c_{0}+\frac{\sigma_{\theta}^{2}\left(\rho_{\theta}^{2}+1\right)}{c_{2}} \\
-\frac{c_{2}}{2} \frac{\sigma_{\theta}^{2}\left(\rho_{\theta}^{2}+1\right)}{c_{2}^{2}}
\end{gathered}
$$

This expression simplifies to: 


$$
E W_{N B P}^{C}=2\left(b_{0}-c_{0}\right)+\frac{\sigma_{\theta}^{2}}{2 c_{2}^{2}}\left(\rho_{\theta}^{2}+2\right)\left(c_{2}-b_{2}\right)
$$

It follows that the advantage of non-bankable prices over non-bankable quantities is

$$
\Delta_{N B P, N B Q}^{C}=\frac{\sigma_{\theta}^{2}}{c_{2}}\left(\rho_{\theta}^{2}+2\right)\left(c_{2}-b_{2}\right)
$$

\section{Bankable quantity}

The firm's problem is

$$
\begin{aligned}
& \max _{q_{1}, q_{2}, B}-C\left(q_{1}, \theta_{1}\right)-C\left(q_{2}, \theta_{2}\right) \\
& \text { s.t. } q_{1}=\tilde{q}_{1}-B, q_{2}=\tilde{q}_{2}+B \\
& \max _{q_{1}, q_{2}, B}-\left[c_{0}+\left(c_{1}+\theta_{1}\right)\left(\tilde{q}_{1}-B-\hat{q}\right)+\frac{c_{2}}{2}\left(\tilde{q}_{1}-B-\hat{q}\right)^{2}+c_{0}+\left(c_{1}+\theta_{2}\right)\left(\tilde{q}_{2}+B-\hat{q}\right)+\right. \\
& \left.\frac{c_{2}}{2}\left(\tilde{q}_{2}+B-\hat{q}\right)^{2}\right]
\end{aligned}
$$

The first-order condition with respect to $B$ yields $B=\frac{\tilde{q}_{1}-\tilde{q}_{2}}{2}+\frac{\theta_{1}-\theta_{2}}{2 c_{2}}$. This implies $q_{1}=\tilde{q}_{1}-$ $B=\frac{\tilde{q}_{1}+\tilde{q}_{2}}{2}-\frac{\theta_{1}-\theta_{2}}{2 c_{2}}$. Similarly, $q_{2}=\tilde{q}_{2}+B=\frac{\tilde{q}_{1}+\tilde{q}_{2}}{2}+\frac{\theta_{1}-\theta_{2}}{2 c_{2}}$. Let $\tilde{q}_{1}+\tilde{q}_{2}=\tilde{q}$. Given this response, the planner sets the quantities $\left\{\tilde{q}_{1}, \tilde{q}_{2}\right\}$ to maximize expected net benefits:

$$
\begin{aligned}
\max _{\tilde{q}} E\left[b_{0}+\right. & \left(b_{1}+\eta_{1}\right)\left(\frac{\tilde{q}_{1}+\tilde{q}_{2}}{2}-\frac{\theta_{1}-\theta_{2}}{2 c_{2}}-\hat{q}\right)-\frac{b_{2}}{2}\left(\frac{\tilde{q}_{1}+\tilde{q}_{2}}{2}-\frac{\theta_{1}-\theta_{2}}{2 c_{2}}-\hat{q}\right)^{2}-c_{0} \\
& -\left(c_{1}+\theta_{1}\right)\left(\frac{\tilde{q}_{1}+\tilde{q}_{2}}{2}-\frac{\theta_{1}-\theta_{2}}{2 c_{2}}-\hat{q}\right)-\frac{c_{2}}{2}\left(\frac{\tilde{q}_{1}+\tilde{q}_{2}}{2}-\frac{\theta_{1}-\theta_{2}}{2 c_{2}}-\hat{q}\right)^{2}+b_{0} \\
& +\left(b_{1}+\eta_{2}\right)\left(\frac{\tilde{q}_{1}+\tilde{q}_{2}}{2}+\frac{\theta_{1}-\theta_{2}}{2 c_{2}}-\hat{q}\right)-\frac{b_{2}}{2}\left(\frac{\tilde{q}_{1}+\tilde{q}_{2}}{2}+\frac{\theta_{1}-\theta_{2}}{2 c_{2}}-\hat{q}\right)^{2}-c_{0} \\
& \left.-\left(c_{1}+\theta_{2}\right)\left(\frac{\tilde{q}_{1}+\tilde{q}_{2}}{2}+\frac{\theta_{1}-\theta_{2}}{2 c_{2}}-\hat{q}\right)-\frac{c_{2}}{2}\left(\frac{\tilde{q}_{1}+\tilde{q}_{2}}{2}+\frac{\theta_{1}-\theta_{2}}{2 c_{2}}-\hat{q}\right)^{2}\right]
\end{aligned}
$$


Using $E\left[\left(\theta_{1}-\theta_{2}\right)^{2}\right]=\sigma_{\theta}^{2}\left(\rho_{\theta}^{2}-2 \rho_{\theta}+2\right) ; E\left[\theta_{1}\left(\theta_{1}-\theta_{2}\right)\right]=\sigma_{\theta}^{2}\left(1-\rho_{\theta}\right) ; E\left[\theta_{2}\left(\theta_{1}-\right.\right.$

$\left.\left.\theta_{2}\right)\right]=\sigma_{\theta}^{2}\left(\rho_{\theta}-\rho_{\theta}^{2}-1\right)$ and taking first order condition of the above expression with respect to $\tilde{q}_{1}+\tilde{q}_{2}$ yields:

$$
\tilde{q}_{1}+\tilde{q}_{2}=2 \hat{q}
$$

and substituting this back into the net benefit expression above gives:

$$
E W_{B Q}^{C}=2\left(b_{0}-c_{0}\right)+\frac{\sigma_{\theta}^{2}}{4 c_{2}^{2}}\left(\rho_{\theta}^{2}-2 \rho_{\theta}+2\right)\left(c_{2}-b_{2}\right)
$$

\section{Bankable price}

The firm faces prices in each period $\tilde{p}_{1}$ and $\tilde{p}_{2}$. The firm's problem is:

$$
\begin{gathered}
\max _{q_{1}, q_{2}, B} \tilde{p}_{1}\left(q_{1}-B\right)-C\left(q_{1}, \theta_{1}\right)+\tilde{p}_{2}\left(q_{2}+B\right)-C\left(q_{2}, \theta_{2}\right) \\
\text { s.t. } q_{1}-B \geq 0, q_{2}+B \geq 0
\end{gathered}
$$

Use the Kuhn-Tucker method to solve this optimization problem with inequality constraints.

$$
\begin{aligned}
& \mathcal{L}\left(q_{1}, q_{2}, B ; \lambda_{1}, \lambda_{2}\right)=\tilde{p}_{1}\left(q_{1}-B\right)-C\left(q_{1}, \theta_{1}\right)+\tilde{p}_{2}\left(q_{2}+B\right)-C\left(q_{2}, \theta_{2}\right)+\lambda_{1}\left(q_{1}-B\right)+ \\
& \lambda_{2}\left(q_{2}+B\right) \\
& \frac{\partial \mathcal{L}}{\partial q_{1}}=\tilde{p}_{1}-C^{\prime}\left(q_{1}, \theta_{1}\right)+\lambda_{1}=0 \\
& \frac{\partial \mathcal{L}}{\partial q_{2}}=\tilde{p}_{2}-C^{\prime}\left(q_{2}, \theta_{2}\right)+\lambda_{2}=0 \\
& \frac{\partial \mathcal{L}}{\partial B}=-\tilde{p}_{1}+\tilde{p}_{2}-\lambda_{1}+\lambda_{2}=0 \\
& \frac{\partial \mathcal{L}}{\partial \lambda_{1}}=q_{1}-B \geq 0, \lambda_{1} \geq 0 \text { and } \lambda_{1}\left(q_{1}-B\right)=0 \\
& \frac{\partial \mathcal{L}}{\partial \lambda_{2}}=q_{2}+B \geq 0, \lambda_{2} \geq 0 \text { and } \lambda_{2}\left(q_{2}+B\right)=0
\end{aligned}
$$

Now consider various cases as in Scenario A:

Case 1: $\lambda_{1}=\lambda_{2}=0$ 
Solving this gives $\tilde{p}_{1}=\tilde{p}_{2}$ and

$$
\begin{aligned}
& q_{1}=\hat{q}+\frac{\tilde{p}_{1}-c_{1}-\theta_{1}}{c_{2}} \\
& q_{2}=\hat{q}+\frac{\tilde{p}_{2}-c_{1}-\theta_{2}}{c_{2}}
\end{aligned}
$$

Case 2: $\lambda_{1}=0, \lambda_{2}>0$

Solving this gives $\tilde{p}_{1}>\tilde{p}_{2}$ and

$$
\begin{aligned}
& q_{1}=\hat{q}+\frac{\tilde{p}_{1}-c_{1}-\theta_{1}}{c_{2}} \\
& q_{2}=\hat{q}+\frac{\tilde{p}_{1}-c_{1}-\theta_{2}}{c_{2}}
\end{aligned}
$$

\section{Case 3: $\lambda_{1}>0, \lambda_{2}=0$}

Solving this gives $\tilde{p}_{2}>\tilde{p}_{1}$ and

$$
\begin{aligned}
& q_{1}=\hat{q}+\frac{\tilde{p}_{2}-c_{1}-\theta_{1}}{c_{2}} \\
& q_{2}=\hat{q}+\frac{\tilde{p}_{2}-c_{1}-\theta_{2}}{c_{2}}
\end{aligned}
$$

\section{Case 4: $\lambda_{1}>0, \lambda_{2}>0$}

This is the trivial case in which both periods' prices are so low as to make the firm never produce in either period, so this case is ignored as in Scenario A.

The planner needs to only choose any one price, say $\tilde{p}$, and the other (lower) price becomes irrelevant. So, the expected net benefits will be: 


$$
\begin{gathered}
\max _{\tilde{p}} E\left[b_{0}+\left(b_{1}+\eta_{1}\right)\left(\frac{\tilde{p}-c_{1}-\theta_{1}}{c_{2}}\right)-\frac{b_{2}}{2}\left(\frac{\tilde{p}-c_{1}-\theta_{1}}{c_{2}}\right)^{2}-c_{0}-\left(c_{1}+\theta_{1}\right)\left(\frac{\tilde{p}-c_{1}-\theta_{1}}{c_{2}}\right)\right. \\
-\frac{c_{2}}{2}\left(\frac{\tilde{p}-c_{1}-\theta_{1}}{c_{2}}\right)^{2}+b_{0}+\left(b_{1}+\eta_{2}\right)\left(\frac{\tilde{p}-c_{1}-\theta_{2}}{c_{2}}\right)-\frac{b_{2}}{2}\left(\frac{\tilde{p}-c_{1}-\theta_{2}}{c_{2}}\right)^{2}-c_{0} \\
\left.-\left(c_{1}+\theta_{2}\right)\left(\frac{\tilde{p}-c_{1}-\theta_{2}}{c_{2}}\right)-\frac{c_{2}}{2}\left(\frac{\tilde{p}-c_{1}-\theta_{2}}{c_{2}}\right)^{2}\right] \\
\max _{\tilde{p}}\left[b_{0}+b_{1}\left(\frac{\tilde{p}-c_{1}}{c_{2}}\right)-\frac{b_{2}}{2}\left(\frac{\tilde{p}-c_{1}}{c_{2}}\right)^{2}-\frac{b_{2}}{2} \frac{\sigma_{\theta}^{2}}{c_{2}^{2}}-c_{0}-c_{1}\left(\frac{\tilde{p}-c_{1}}{c_{2}}\right)+\frac{\sigma_{\theta}^{2}}{c_{2}}-\frac{c_{2}}{2}\left(\frac{\tilde{p}-c_{1}}{c_{2}}\right)^{2}\right. \\
-\frac{c_{2}}{2} \frac{\sigma_{\theta}^{2}}{c_{2}^{2}}+b_{0}+b_{1}\left(\frac{\tilde{p}-c_{1}}{c_{2}}\right)^{2}-\frac{b_{2}}{2}\left(\frac{\tilde{p}-c_{1}}{c_{2}}\right)^{2}-\frac{b_{2}}{2} \frac{\sigma_{\theta}^{2}\left(\rho_{\theta}^{2}+1\right)}{c_{2}^{2}}-c_{0}-c_{1}\left(\frac{\tilde{p}-c_{1}}{c_{2}}\right) \\
\left.+\frac{\sigma_{\theta}^{2}\left(\rho_{\theta}^{2}+1\right)}{c_{2}}-\frac{c_{2}}{2}\left(\frac{\tilde{p}-c_{1}}{c_{2}}\right)^{2}-\frac{c_{2}}{2} \frac{\sigma_{\theta}^{2}\left(\rho_{\theta}^{2}+1\right)}{c_{2}^{2}}\right]
\end{gathered}
$$

The first-order condition for choice of $\tilde{p}$ is

$$
\frac{b_{1}}{c_{2}}-\frac{b_{2}}{c_{2}^{2}}\left(\tilde{p}-c_{1}\right)-\frac{c_{1}}{c_{2}}-\frac{1}{c_{2}}\left(\tilde{p}-c_{1}\right)+\frac{b_{1}}{c_{2}}-\frac{b_{2}}{c_{2}^{2}}\left(\tilde{p}-c_{1}\right)-\frac{c_{1}}{c_{2}}-\frac{1}{c_{2}}\left(\tilde{p}-c_{1}\right)=0
$$

This gives:

$$
\tilde{p}=c_{1}
$$

Therefore, the maximized expected welfare expression becomes:

$$
E W_{B P}^{C}=2\left(b_{0}-c_{0}\right)+\frac{\sigma_{\theta}^{2}}{2 c_{2}^{2}}\left(\rho_{\theta}^{2}+2\right)\left(c_{2}-b_{2}\right)
$$

\section{A.1.D Scenario D}

First best

Since the shock values are the same as in scenario $\mathrm{C}$, the first-best solution is identical also: $q_{1}=\hat{q}+\frac{\eta_{1}-\theta_{1}}{b_{2}+c_{2}}$ and $q_{2}=\hat{q}+\frac{\eta_{2}-\theta_{2}}{b_{2}+c_{2}}$.

Non-bankable quantity 
The firm has no choice but to produce $\left\{\tilde{q}_{1}, \tilde{q}_{2}\right\}$. The planner's problem is now a twoperiod problem, since it can update its quantity policy after the first period. The second-period problem is:

$$
\max _{\tilde{q}_{2}} E\left[b_{0}+\left(b_{1}+\eta_{2}\right)\left(\tilde{q}_{2}-\hat{q}\right)-\frac{b_{2}}{2}\left(\tilde{q}_{2}-\hat{q}\right)^{2}-c_{0}-\left(c_{1}+\theta_{2}\right)\left(\tilde{q}_{2}-\hat{q}\right)-\frac{c_{2}}{2}\left(\tilde{q}_{2}-\hat{q}\right)^{2}\right]
$$

Using $E\left[\theta_{2} \mid \theta_{1}\right]=\rho_{\theta} \theta_{1}$ and $E\left[\eta_{2} \mid \eta_{1}\right]=\rho_{\eta} \eta_{1}$, the first-order condition yields:

$$
\tilde{q}_{2}=\hat{q}+\frac{\rho_{\eta} \eta_{1}-\rho_{\theta} \theta_{1}}{b_{2}+c_{2}}
$$

The first period problem is:

$$
\begin{gathered}
\max _{\tilde{q}_{1}} E\left[b_{0}+\left(b_{1}+\eta_{1}\right)\left(\tilde{q}_{1}-\hat{q}\right)-\frac{b_{2}}{2}\left(\tilde{q}_{1}-\hat{q}\right)^{2}-c_{0}-\left(c_{1}+\theta_{1}\right)\left(\tilde{q}_{1}-\hat{q}\right)-\frac{c_{2}}{2}\left(\tilde{q}_{1}-\hat{q}\right)^{2}+b_{0}\right. \\
\left.+\left(b_{1}+\eta_{2}\right)\left(\tilde{q}_{2}-\hat{q}\right)-\frac{b_{2}}{2}\left(\tilde{q}_{2}-\hat{q}\right)^{2}-c_{0}-\left(c_{1}+\theta_{2}\right)\left(\tilde{q}_{2}-\hat{q}\right)-\frac{c_{2}}{2}\left(\tilde{q}_{2}-\hat{q}\right)^{2}\right] \\
\max _{\tilde{q}_{1}} E\left[b_{0}+\left(b_{1}+\eta_{1}\right)\left(\tilde{q}_{1}-\hat{q}\right)-\frac{b_{2}}{2}\left(\tilde{q}_{1}-\hat{q}\right)^{2}-c_{0}-\left(c_{1}+\theta_{1}\right)\left(\tilde{q}_{1}-\hat{q}\right)-\frac{c_{2}}{2}\left(\tilde{q}_{1}-\hat{q}\right)^{2}+b_{0}\right. \\
+\left(b_{1}+\eta_{2}\right)\left(\frac{\rho_{\eta} \eta_{1}-\rho_{\theta} \theta_{1}}{b_{2}+c_{2}}\right)-\frac{b_{2}}{2}\left(\frac{\rho_{\eta} \eta_{1}-\rho_{\theta} \theta_{1}}{b_{2}+c_{2}}\right)^{2}-c_{0} \\
\left.-\left(c_{1}+\theta_{2}\right)\left(\frac{\rho_{\eta} \eta_{1}-\rho_{\theta} \theta_{1}}{b_{2}+c_{2}}\right)-\frac{c_{2}}{2}\left(\frac{\rho_{\eta} \eta_{1}-\rho_{\theta} \theta_{1}}{b_{2}+c_{2}}\right)^{2}\right]
\end{gathered}
$$

The first-order condition with respect to $\tilde{q}_{1}$ is

$$
b_{1}-b_{2}\left(\tilde{q}_{1}-\hat{q}\right)-c_{1}-c_{2}\left(\tilde{q}_{1}-\hat{q}\right)=0
$$

Therefore,

$$
\tilde{q}_{1}=\hat{q}
$$

Substituting these two solutions for $\tilde{q}_{1}$ and $\tilde{q}_{2}$ into the expresstion for expected welfare (note that all of the first-period terms with $\tilde{q}_{1}-\hat{q}$ become zero) yields 


$$
\begin{aligned}
E W_{N B Q}^{B}= & b_{0}-c_{0}+b_{0}+\frac{\rho_{\eta}{ }^{2} \sigma_{\eta}^{2}}{b_{2}+c_{2}}-\frac{b_{2}}{2\left(b_{2}+c_{2}\right)^{2}}\left[\rho_{\eta}^{2} \sigma_{\eta}^{2}+\rho_{\theta}^{2} \sigma_{\theta}^{2}\right]-c_{0}+\frac{\rho_{\theta}^{2} \sigma_{\theta}^{2}}{\left(b_{2}+c_{2}\right)} \\
& -\frac{c_{2}}{2\left(b_{2}+c_{2}\right)^{2}}\left[\rho_{\eta}^{2} \sigma_{\eta}^{2}+\rho_{\theta}^{2} \sigma_{\theta}^{2}\right] \\
= & 2\left(b_{0}-c_{0}\right)+\frac{1}{2\left(b_{2}+c_{2}\right)}\left[\rho_{\eta}^{2} \sigma_{\eta}^{2}+\rho_{\theta}^{2} \sigma_{\theta}^{2}\right]
\end{aligned}
$$

\section{Non-bankable price}

Given a set of policies $\left\{\tilde{p}_{1}, \tilde{p}_{2}\right\}$, the firm chooses $q_{1}=\hat{q}+\frac{\tilde{p}_{1}-c_{1}-\theta_{1}}{c_{2}}$ and $q_{2}=\hat{q}+$ $\frac{\tilde{p}_{2}-c_{1}-\theta_{2}}{c_{2}}$. Given this response, the planner's second period problem is as follows:

$$
\begin{gathered}
\max _{\tilde{p}_{2}}\left[b_{0}+\left(b_{1}+\eta_{2}\right)\left(q_{2}-\hat{q}\right)-\frac{b_{2}}{2}\left(q_{2}-\hat{q}\right)^{2}-c_{0}-\left(c_{1}+\theta_{2}\right)\left(q_{2}-\hat{q}\right)-\frac{c_{2}}{2}\left(q_{2}-\hat{q}\right)^{2}\right] \\
=\max _{\tilde{p}_{2}}\left[b_{0}+\left(b_{1}+\eta_{2}\right)\left(\frac{\tilde{p}_{2}-c_{1}-\theta_{2}}{c_{2}}\right)-\frac{b_{2}}{2}\left(\frac{\tilde{p}_{2}-c_{1}-\theta_{2}}{c_{2}}\right)^{2}-c_{0}-\left(c_{1}+\theta_{2}\right)\left(\frac{\tilde{p}_{2}-c_{1}-\theta_{2}}{c_{2}}\right)\right. \\
-\frac{c_{2}}{2}\left(\frac{\tilde{p}_{2}-c_{1}-\theta_{2}}{c_{2}}\right)^{2}
\end{gathered}
$$

Using $E\left[\eta_{2} \theta_{2} \mid \eta_{1}, \theta_{1}\right]=\rho_{\theta} \theta_{1} \rho_{\eta} \eta_{1}$ and $E\left[\theta_{2}{ }^{2} \mid \eta_{1}, \theta_{1}\right]=\rho_{\theta} \theta_{1}^{2}+\sigma_{\theta}^{2}$; and solving for the firstorder condition with respect to $\tilde{p}_{2}$ yields:

$$
\tilde{p}_{2}=c_{1}+\frac{\rho_{\theta} \theta_{1} b_{2}+\rho_{\eta} \eta_{1} c_{2}}{b_{2}+c_{2}}
$$

Firm's $q_{2}$ is:

$$
q_{2}=\hat{q}+\frac{1}{c_{2}}\left(\frac{\rho_{\theta} \theta_{1} b_{2}+\rho_{\eta} \eta_{1} c_{2}}{b_{2}+c_{2}}\right)-\frac{\theta_{2}}{c_{2}}
$$

Moving to the planner's first-period problem: 


$$
\begin{gathered}
\max _{\tilde{p}_{1}} E\left[b_{0}+\left(b_{1}+\eta_{1}\right)\left(\frac{\tilde{p}_{1}-c_{1}-\theta_{1}}{c_{2}}\right)-\frac{b_{2}}{2}\left(\frac{\tilde{p}_{1}-c_{1}-\theta_{1}}{c_{2}}\right)^{2}-c_{0}-\left(c_{1}+\theta_{1}\right)\left(\frac{\tilde{p}_{1}-c_{1}-\theta_{1}}{c_{2}}\right)\right. \\
-\frac{c_{2}}{2}\left(\frac{\tilde{p}_{1}-c_{1}-\theta_{1}}{c_{2}}\right)^{2}+b_{0}+\left(b_{1}+\eta_{2}\right)\left(\frac{\tilde{p}_{2}-c_{1}-\theta_{2}}{c_{2}}\right)-\frac{b_{2}}{2}\left(\frac{\tilde{p}_{2}-c_{1}-\theta_{2}}{c_{2}}\right)^{2} \\
\left.-c_{0}-\left(c_{1}+\theta_{2}\right)\left(\frac{\tilde{p}_{2}-c_{1}-\theta_{2}}{c_{2}}\right)-\frac{c_{2}}{2}\left(\frac{\tilde{p}_{2}-c_{1}-\theta_{2}}{c_{2}}\right)^{2}\right]
\end{gathered}
$$

Taking the above expectation and then solving for first order condition with respect to $\tilde{p}_{1}$ yields:

$$
\tilde{p}_{1}=c_{1}
$$

The firm's $q_{1}$ is

$$
q_{1}=\hat{q}-\frac{\theta_{1}}{c_{2}}
$$

Substituting the firm's choices for $q_{1}$ and $q_{2}$ into the expected welfare function gives:

$$
\begin{gathered}
E W_{N B P}^{D}=E\left[b_{0}+\left(b_{1}+\eta_{1}\right)\left(-\frac{\theta_{1}}{c_{2}}\right)-\frac{b_{2}}{2}\left(-\frac{\theta_{1}}{c_{2}}\right)^{2}-c_{0}-\left(c_{1}+\theta_{1}\right)\left(-\frac{\theta_{1}}{c_{2}}\right)-\frac{c_{2}}{2}\left(-\frac{\theta_{1}}{c_{2}}\right)^{2}+b_{0}\right. \\
\left.+\left(b_{1}+\eta_{2}\right)\left(q_{2}-\hat{q}\right)-\frac{b_{2}}{2}\left(q_{2}-\hat{q}\right)^{2}-c_{0}-\left(c_{1}+\theta_{2}\right)\left(q_{2}-\hat{q}\right)-\frac{c_{2}}{2}\left(q_{2}-\hat{q}\right)^{2}\right] \\
=b_{0}-\frac{b_{2}+c_{2}}{2 c_{2}{ }^{2}} \sigma_{\theta}^{2}-c_{0}+\frac{\sigma_{\theta}^{2}}{c_{2}}-\frac{c_{2}}{2 c_{2}{ }^{2}} \sigma_{\theta}^{2}+b_{0}+E\left[\eta_{2}\left(q_{2}-\hat{q}\right)\right]-\frac{b_{2}+c_{2}}{2} E\left[\left(q_{2}-\hat{q}\right)^{2}\right]-c_{0}- \\
E\left[\theta_{2}\left(q_{2}-\hat{q}\right)\right]
\end{gathered}
$$

Using the following expressions to simplify $E W_{N B P}^{D}: E\left[\left(q_{2}-\hat{q}\right)\right]=0 ; E\left[\eta_{2}\left(q_{2}-\hat{q}\right)\right]=\frac{\rho_{\eta}^{2} \sigma_{\eta}^{2}}{b_{2}+c_{2}}$;

$E\left[\theta_{2}\left(q_{2}-\hat{q}\right)\right]=-\sigma_{\theta}^{2}\left(\frac{\rho_{\theta}^{2}}{\left(b_{2}+c_{2}\right)}+\frac{1}{c_{2}}\right) ; E\left[\left(q_{2}-\hat{q}\right)^{2}\right]=\left[\frac{\sigma_{\theta}^{2}}{c_{2}^{2}}\left(\rho_{\theta}^{2}\left(\frac{b_{2}}{b_{2}+c_{2}}\right)^{2}+\rho_{\theta}^{2}+1-\right.\right.$ $\left.\left.2 \rho_{\theta}{ }^{2} \frac{b_{2}}{b_{2}+c_{2}}\right)+\frac{\rho_{\eta}{ }^{2}}{\left(b_{2}+c_{2}\right)} \sigma_{\eta}^{2}\right]$, we get 


$$
\begin{aligned}
E W_{N B P}^{D}=b_{0} & -\frac{b_{2}+c_{2}}{2 c_{2}{ }^{2}} \sigma_{\theta}^{2}-c_{0}+\frac{\sigma_{\theta}^{2}}{c_{2}}-\frac{c_{2}}{2 c_{2}{ }^{2}} \sigma_{\theta}^{2}+b_{0}+\frac{\rho_{\eta}{ }^{2} \sigma_{\eta}^{2}}{b_{2}+c_{2}} \\
& -\frac{b_{2}+c_{2}}{2}\left[\frac{\sigma_{\theta}^{2}}{c_{2}{ }^{2}}\left(\rho_{\theta}{ }^{2}\left(\frac{b_{2}}{b_{2}+c_{2}}\right)^{2}+\rho_{\theta}{ }^{2}+1-2 \rho_{\theta}{ }^{2} \frac{b_{2}}{b_{2}+c_{2}}\right)+\frac{\rho_{\eta}{ }^{2}}{\left(b_{2}+c_{2}\right)} \sigma_{\eta}^{2}\right] \\
& -c_{0}-\left[-\sigma_{\theta}^{2}\left(\frac{\rho_{\theta}{ }^{2}}{\left(b_{2}+c_{2}\right)}+\frac{1}{c_{2}}\right)\right]
\end{aligned}
$$

On simplification, this expression becomes:

$$
E W_{N B P}^{B}=2\left(b_{0}-c_{0}\right)+\frac{1}{2\left(b_{2}+c_{2}\right)}\left[\rho_{\eta}{ }^{2} \sigma_{\eta}^{2}+\rho_{\theta}^{2} \sigma_{\theta}^{2}\right]+\frac{\sigma_{\theta}^{2}}{c_{2}{ }^{2}}\left(c_{2}-b_{2}\right)
$$

\section{Bankable quantity}

The firm's problem here is the same as the bankable quantity policy under scenario C.

Therefore, $q_{1}=\frac{\tilde{q}_{1}+\tilde{q}_{2}}{2}-\frac{\theta_{1}-\theta_{2}}{2 c_{2}}$ and $q_{2}=\frac{\tilde{q}_{1}+\tilde{q}_{2}}{2}+\frac{\theta_{1}-\theta_{2}}{2 c_{2}}$. Let $\frac{\tilde{q}_{1}+\tilde{q}_{2}}{2} \equiv \tilde{q}$. Given this response, the planner's problem becomes

$$
\begin{aligned}
& \begin{aligned}
\max _{\tilde{q}} E\left[b_{0}+(\right. & \left.b_{1}+\eta_{1}\right)\left(q_{1}-\hat{q}\right)-\frac{b_{2}}{2}\left(q_{1}-\hat{q}\right)^{2}-c_{0}-\left(c_{1}+\theta_{1}\right)\left(q_{1}-\hat{q}\right)-\frac{c_{2}}{2}\left(q_{1}-\hat{q}\right)^{2}+b_{0} \\
& +\left(b_{1}+\eta_{2}\right)\left(q_{2}-\hat{q}\right)-\frac{b_{2}}{2}\left(q_{2}-\hat{q}\right)^{2}-c_{0}-\left(c_{1}+\theta_{2}\right)\left(q_{2}-\hat{q}\right) \\
& \left.\left.-\frac{c_{2}}{2}\left(q_{2}-\hat{q}\right)^{2} \mid \theta_{1}, \eta_{1}\right]\right]
\end{aligned} \\
& \text { s.t. } q_{1}=\frac{\tilde{q}_{1}+\tilde{q}_{2}}{2}-\frac{\theta_{1}-\theta_{2}}{2 c_{2}} \text { and } q_{2}=\frac{\tilde{q}_{1}+\tilde{q}_{2}}{2}+\frac{\theta_{1}-\theta_{2}}{2 c_{2}}
\end{aligned}
$$

Consider the following expectations:

$$
\begin{gathered}
E\left(q_{1}-\hat{q}\right)=\tilde{q}-\frac{\theta_{1}-\rho_{\theta} \theta_{1}}{2 c_{2}}-\hat{q} \\
E\left(q_{1}-\hat{q}\right)^{2}=\left(\tilde{q}-\frac{\theta_{1}}{2 c_{2}}-\hat{q}\right)^{2}+\left(\tilde{q}-\frac{\theta_{1}}{2 c_{2}}-\hat{q}\right) \frac{\rho_{\theta} \theta_{1}}{c_{2}}+\frac{\rho_{\theta}{ }^{2} \theta_{1}{ }^{2}+\sigma_{\theta}^{2}}{4 c_{2}{ }^{2}}
\end{gathered}
$$




$$
\begin{gathered}
E\left(q_{2}-\hat{q}\right)=\tilde{q}+\frac{\theta_{1}-\rho_{\theta} \theta_{1}}{2 c_{2}}-\hat{q} \\
E\left[\eta_{2}\left(q_{2}-\hat{q}\right)\right]=\rho_{\eta} \eta_{1} \tilde{q}-\frac{\rho_{\eta} \eta_{1} \rho_{\theta} \theta_{1}}{2 c_{2}}-\rho_{\eta} \eta_{1} \hat{q} \\
E\left[\theta_{2}\left(q_{2}-\hat{q}\right)\right]=\rho_{\theta} \theta_{1} \tilde{q}-\frac{\rho_{\theta} \theta_{1}{ }^{2}-\left(\rho_{\theta}{ }^{2} \theta_{1}{ }^{2}+\sigma_{\theta}^{2}\right)}{2 c_{2}}-\rho_{\theta} \theta_{1} \hat{q} \\
E\left(q_{2}-\hat{q}\right)^{2}=\left(\tilde{q}+\frac{\theta_{1}}{2 c_{2}}-\hat{q}\right)^{2}+\left(\tilde{q}+\frac{\theta_{1}}{2 c_{2}}-\hat{q}\right) \frac{\rho_{\theta} \theta_{1}}{c_{2}}+\frac{\rho_{\theta}{ }^{2} \theta_{1}{ }^{2}+\sigma_{\theta}^{2}}{4 c_{2}{ }^{2}}
\end{gathered}
$$

Using these expectation expressions to simplify the planner's optimization problem and then taking the first-order condition yields:

$$
\tilde{q}-\hat{q}=\frac{\eta_{1}\left(1+\rho_{\eta}\right)-\theta_{1}\left(1+\rho_{\theta}\right)}{2\left(b_{2}+c_{2}\right)}
$$

or,

$$
\tilde{q}-\hat{q}=\frac{\eta_{1}-\theta_{1}}{2\left(b_{2}+c_{2}\right)}+\frac{\rho_{\eta} \eta_{1}-\rho_{\theta} \theta_{1}}{2\left(b_{2}+c_{2}\right)}
$$

This implies:

$$
\begin{aligned}
& q_{1}=\hat{q}+\frac{\eta_{1}\left(1+\rho_{\eta}\right)-\theta_{1}\left(1+\rho_{\theta}\right)}{2\left(b_{2}+c_{2}\right)}-\frac{\theta_{1}-\theta_{2}}{2 c_{2}} \\
& q_{2}=\hat{q}+\frac{\eta_{1}\left(1+\rho_{\eta}\right)-\theta_{1}\left(1+\rho_{\theta}\right)}{2\left(b_{2}+c_{2}\right)}+\frac{\theta_{1}-\theta_{2}}{2 c_{2}}
\end{aligned}
$$

Substituting these values into expected welfare function: 


$$
\begin{aligned}
E W_{B Q}^{D}=E\left[b_{0}\right. & +\left(b_{1}+\eta_{1}\right)\left(q_{1}-\hat{q}\right)-\frac{b_{2}}{2}\left(q_{1}-\hat{q}\right)^{2}-c_{0}-\left(c_{1}+\theta_{1}\right)\left(q_{1}-\hat{q}\right)-\frac{c_{2}}{2}\left(q_{1}-\hat{q}\right)^{2} \\
& +b_{0}+\left(b_{1}+\eta_{2}\right)\left(q_{2}-\hat{q}\right)-\frac{b_{2}}{2}\left(q_{2}-\hat{q}\right)^{2}-c_{0}-\left(c_{1}+\theta_{2}\right)\left(q_{2}-\hat{q}\right) \\
& \left.-\frac{c_{2}}{2}\left(q_{2}-\hat{q}\right)^{2} \mid \theta_{1}, \eta_{1}\right]
\end{aligned}
$$

Solving this by substituting optimal $q_{1}$ and $q_{2}$, and then simplifying it yields:

$$
E W_{B Q}^{D}=2\left(b_{0}-c_{0}\right)+\frac{1}{4} \frac{\left(1+\rho_{\eta}\right)^{2} \sigma_{\eta}^{2}+\left(1+\rho_{\theta}\right)^{2} \sigma_{\theta}^{2}}{b_{2}+c_{2}}+\frac{\sigma_{\theta}^{2}}{4 c_{2}^{2}}\left(c_{2}-b_{2}\right)\left(2+\rho_{\theta}^{2}-2 \rho_{\theta}\right)
$$

\section{Bankable price}

The firm's response to this policy is the same as its response under scenario C:

$$
\begin{aligned}
& q_{1}=\hat{q}+\frac{\max \left\{\tilde{p}_{1}, \tilde{p}_{2}\right\}-c_{1}-\theta_{1}}{c_{2}} \\
& q_{2}=\hat{q}+\frac{\max \left\{\tilde{p}_{1}, \tilde{p}_{2}\right\}-c_{1}-\theta_{2}}{c_{2}}
\end{aligned}
$$

Assuming that $\tilde{p}_{2}$ is the binding price, the planner's second-period problem is

$$
\begin{gathered}
\max _{\tilde{p}_{2}} E\left[b_{0}+\left(b_{1}+\eta_{2}\right)\left(q_{2}-\hat{q}\right)-\frac{b_{2}}{2}\left(q_{2}-\hat{q}\right)^{2}-c_{0}-\left(c_{1}+\theta_{2}\right)\left(q_{2}-\hat{q}\right)-\frac{c_{2}}{2}\left(q_{2}-\hat{q}\right)^{2}\right] \\
\text { s.t. } q_{2}=\hat{q}+\frac{\max \left\{\tilde{p}_{1}, \tilde{p}_{2}\right\}-c_{1}-\theta_{2}}{c_{2}} \\
=\max _{\tilde{p}_{2}}\left[b_{0}+\left(b_{1}+\eta_{2}\right)\left(\frac{\tilde{p}_{2}-c_{1}-\theta_{2}}{c_{2}}\right)-\frac{b_{2}}{2}\left(\frac{\tilde{p}_{2}-c_{1}-\theta_{2}}{c_{2}}\right)^{2}-c_{0}-\left(c_{1}+\theta_{2}\right)\left(\frac{\tilde{p}_{2}-c_{1}-\theta_{2}}{c_{2}}\right)\right. \\
\left.-\frac{c_{2}}{2}\left(\frac{\tilde{p}_{2}-c_{1}-\theta_{2}}{c_{2}}\right)^{2} \mid \theta_{1}, \eta_{1}\right]
\end{gathered}
$$

The first-order condition with respect to $\tilde{p}_{2}$ yields

$$
\tilde{p}_{2}=c_{1}+\frac{\rho_{\theta} \theta_{1} b_{2}+\rho_{\eta} \eta_{1} c_{2}}{b_{2}+c_{2}}
$$

Firm's $q_{2}$ is: 


$$
q_{2}=\hat{q}+\frac{1}{c_{2}}\left(\frac{\rho_{\theta} \theta_{1} b_{2}+\rho_{\eta} \eta_{1} c_{2}}{b_{2}+c_{2}}\right)-\frac{\theta_{2}}{c_{2}}
$$

Planner will choose $\tilde{p}$ to maximize sum of welfare of both periods. This implies:

$$
\begin{aligned}
\max _{\tilde{p}} E\left[b_{0}+(\right. & \left.b_{1}+\eta_{1}\right)\left(q_{1}-\hat{q}\right)-\frac{b_{2}}{2}\left(q_{1}-\hat{q}\right)^{2}-c_{0}-\left(c_{1}+\theta_{1}\right)\left(q_{1}-\hat{q}\right)-\frac{c_{2}}{2}\left(q_{1}-\hat{q}\right)^{2}+b_{0} \\
& +\left(b_{1}+\eta_{2}\right)\left(q_{2}-\hat{q}\right)-\frac{b_{2}}{2}\left(q_{2}-\hat{q}\right)^{2}-c_{0}-\left(c_{1}+\theta_{2}\right)\left(q_{2}-\hat{q}\right) \\
& \left.\left.-\frac{c_{2}}{2}\left(q_{2}-\hat{q}\right)^{2} \mid \theta_{1}, \eta_{1}\right]\right]
\end{aligned}
$$

s.t.

$$
\begin{aligned}
& q_{1}=\hat{q}+\frac{\tilde{p}-c_{1}-\theta_{1}}{c_{2}} \\
& q_{2}=\hat{q}+\frac{\tilde{p}-c_{1}-\theta_{2}}{c_{2}}
\end{aligned}
$$

On solving this, we get:

$$
\tilde{p}=c_{1}+\frac{\left(1+\rho_{\theta}\right) \theta_{1} b_{2}+\left(1+\rho_{\eta}\right) \eta_{1} c_{2}}{2\left(b_{2}+c_{2}\right)}
$$

and subsequently,

$$
\begin{aligned}
& q_{1}=\hat{q}+\frac{1}{2 c_{2}}\left(\frac{\left(1+\rho_{\theta}\right) \theta_{1} b_{2}+\left(1+\rho_{\eta}\right) \eta_{1} c_{2}}{b_{2}+c_{2}}\right)-\frac{\theta_{1}}{c_{2}} \\
& q_{2}=\hat{q}+\frac{1}{2 c_{2}}\left(\frac{\left(1+\rho_{\theta}\right) \theta_{1} b_{2}+\left(1+\rho_{\eta}\right) \eta_{1} c_{2}}{b_{2}+c_{2}}\right)-\frac{\theta_{2}}{c_{2}}
\end{aligned}
$$

Consider:

$$
\begin{aligned}
& E\left[\left(q_{1}-\hat{q}\right)\right]=E\left[\left(q_{2}-\hat{q}\right)\right]=0 ; E\left[\eta_{1}\left(q_{1}-\hat{q}\right)\right]=\frac{\left(1+\rho_{\eta}\right) \sigma_{\eta}^{2}}{2\left(b_{2}+c_{2}\right)} ; E\left[\theta_{1}\left(q_{1}-\hat{q}\right)\right]=\frac{1}{c_{2}}\left[\frac{b_{2}\left(1+\rho_{\theta}\right)}{2\left(b_{2}+c_{2}\right)}-\right. \\
& 1] \sigma_{\theta}^{2} ; E\left[\left(q_{1}-\hat{q}\right)^{2}\right]=\left[\frac{\sigma_{\theta}^{2}}{c_{2}{ }^{2}}\left(\frac{b_{2}\left(1+\rho_{\theta}\right)}{2\left(b_{2}+c_{2}\right)}-1\right)^{2}+\left(\frac{1+\rho_{\eta}}{2\left(b_{2}+c_{2}\right)}\right)^{2} \sigma_{\eta}^{2}\right] ; E\left[\eta_{2}\left(q_{2}-\hat{q}\right)\right]=\frac{\left(1+\rho_{\eta}\right) \rho_{\eta} \sigma_{\eta}^{2}}{2\left(b_{2}+c_{2}\right)}
\end{aligned}
$$




$$
\begin{aligned}
& E\left[\theta_{2}\left(q_{2}-\hat{q}\right)\right]=\frac{1}{c_{2}}\left[\frac{b_{2}\left(1+\rho_{\theta}\right) \rho_{\theta}}{2\left(b_{2}+c_{2}\right)}-\left(\rho_{\theta}^{2}+1\right)\right] \sigma_{\theta}^{2} ; E\left[\left(q_{2}-\hat{q}\right)^{2}\right]=\left[\frac { \sigma _ { \theta } ^ { 2 } } { c _ { 2 } ^ { 2 } } \left(\left(1+\rho_{\theta}\right)^{2}\left(\frac{b_{2}}{2\left(b_{2}+c_{2}\right)}\right)^{2}+\right.\right. \\
& \left.\left.\left(\rho_{\theta}^{2}+1\right)-\left(1+\rho_{\theta}\right) \rho_{\theta} \frac{b_{2}}{b_{2}+c_{2}}\right)+\left(\frac{\left(1+\rho_{\eta}\right)}{2\left(b_{2}+c_{2}\right)}\right)^{2} \sigma_{\eta}^{2}\right]
\end{aligned}
$$

Using these above expressions and the optimal quantities, we can solve for planner's maximized expected welfare expression and get:

$$
\begin{aligned}
E W_{B P}^{D}=2\left(b_{0}-c_{0}\right) & +\frac{1}{4} \frac{\left(1+\rho_{\eta}\right)^{2} \sigma_{\eta}^{2}}{\left(b_{2}+c_{2}\right)}+\left[\frac{2+\rho_{\theta}{ }^{2}}{2\left(b_{2}+c_{2}\right)}+\left(\frac{b_{2}^{2}}{4 c_{2}^{2}\left(b_{2}+c_{2}\right)}\right)\left(-\rho_{\theta}^{2}+2 \rho_{\theta}-3\right)\right] \sigma_{\theta}^{2} \\
=2\left(b_{0}-c_{0}\right) & +\frac{\left(1+\rho_{\eta}\right)^{2} \sigma_{\eta}^{2}}{4\left(b_{2}+c_{2}\right)}+\left(1+2 \rho_{\theta}+\rho_{\theta}^{2}+3-2 \rho_{\theta}+\rho_{\theta}{ }^{2}\right) \frac{\sigma_{\theta}^{2}}{4\left(b_{2}+c_{2}\right)} \\
& +\left(\frac{b_{2}^{2}}{4 c_{2}^{2}\left(b_{2}+c_{2}\right)}\right)\left(-\rho_{\theta}^{2}+2 \rho_{\theta}-3\right) \sigma_{\theta}^{2} \\
=2\left(b_{0}-c_{0}\right) & +\frac{\left(1+\rho_{\eta}\right)^{2} \sigma_{\eta}^{2}+\left(1+\rho_{\theta}\right)^{2} \sigma_{\theta}^{2}}{4\left(b_{2}+c_{2}\right)}+\left(3-2 \rho_{\theta}+\rho_{\theta}^{2}\right) \frac{\sigma_{\theta}^{2}}{4\left(b_{2}+c_{2}\right)} \\
& +\left(\frac{b_{2}^{2}}{4 c_{2}^{2}\left(b_{2}+c_{2}\right)}\right)\left(-\rho_{\theta}^{2}+2 \rho_{\theta}-3\right) \sigma_{\theta}^{2}
\end{aligned}
$$

This simplifies to:

$$
E W_{B P}^{D}=2\left(b_{0}-c_{0}\right)+\frac{1}{4} \frac{\left(1+\rho_{\eta}\right)^{2} \sigma_{\eta}^{2}+\left(1+\rho_{\theta}\right)^{2} \sigma_{\theta}^{2}}{b_{2}+c_{2}}+\frac{\sigma_{\theta}^{2}}{4 c_{2}^{2}}\left(c_{2}-b_{2}\right)\left(3+\rho_{\theta}^{2}-2 \rho_{\theta}\right)
$$

Let us now comment on the sign of $\Delta_{B P, N B P}^{D}=E W_{B P}^{D}-E W_{N B P}^{D}$ (Equation 25)

$$
\begin{aligned}
& \Delta_{B P, N B P}^{D}=\frac{\sigma_{\theta}^{2}}{4 c_{2}^{2}}\left(c_{2}-b_{2}\right)\left(-1+\rho_{\theta}^{2}-2 \rho_{\theta}\right)+\frac{1}{4} \frac{\sigma_{\eta}^{2}\left(\left(1+\rho_{\eta}\right)^{2}-2 \rho_{\eta}^{2}\right)+\sigma_{\theta}^{2}\left(\left(1+\rho_{\theta}\right)^{2}-2 \rho_{\theta}^{2}\right)}{b_{2}+c_{2}} \\
& =\frac{\sigma_{\theta}^{2}\left(c_{2}^{2}-b_{2}^{2}\right)\left(-1+\rho_{\theta}^{2}-2 \rho_{\theta}\right)+c_{2}^{2} \sigma_{\eta}^{2}\left(\left(1+\rho_{\eta}\right)^{2}-2 \rho_{\eta}^{2}\right)+c_{2}^{2} \sigma_{\theta}^{2}\left(\left(1+\rho_{\theta}\right)^{2}-2 \rho_{\theta}^{2}\right)}{4 c_{2}^{2}\left(b_{2}+c_{2}\right)} \\
& =\frac{\sigma_{\theta}^{2}\left(c_{2}^{2}-b_{2}^{2}\right)\left(-1+\rho_{\theta}^{2}-2 \rho_{\theta}\right)+c_{2}^{2} \sigma_{\eta}^{2}\left(1-\rho_{\eta}^{2}+2 \rho_{\eta}\right)+c_{2}^{2} \sigma_{\theta}^{2}\left(1-\rho_{\theta}^{2}+2 \rho_{\theta}\right)}{4 c_{2}^{2}\left(b_{2}+c_{2}\right)}
\end{aligned}
$$




$$
\begin{aligned}
& =\frac{\sigma_{\theta}^{2}\left(-1+\rho_{\theta}^{2}-2 \rho_{\theta}\right)\left(c_{2}^{2}-b_{2}^{2}-c_{2}^{2}\right)+c_{2}^{2} \sigma_{\eta}^{2}\left(1-\rho_{\eta}^{2}+2 \rho_{\eta}\right)}{4 c_{2}^{2}\left(b_{2}+c_{2}\right)} \\
& =\frac{\sigma_{\theta}^{2}\left(-1+\rho_{\theta}^{2}-2 \rho_{\theta}\right)\left(-b_{2}^{2}\right)+c_{2}^{2} \sigma_{\eta}^{2}\left(1-\rho_{\eta}^{2}+2 \rho_{\eta}\right)}{4 c_{2}^{2}\left(b_{2}+c_{2}\right)}
\end{aligned}
$$

For our considered parametric values, we can check that $-1+\rho_{\theta}^{2}-2 \rho_{\theta}<0$ and $1-\rho_{\eta}^{2}+$ $2 \rho_{\eta}>0$. Therefore, this above expression is positive, i.e.,

$$
\Delta_{B P, N B P}^{D}=E W_{B P}^{D}-E W_{N B P}^{D}>0
$$

So, BP always dominates NBP irrespective of the parametric values. 